\title{
PDHL-EDAS METHOD FOR MULTIPLE ATTRIBUTE GROUP DECISION MAKING AND ITS APPLICATION TO 3D PRINTER SELECTION
}

\author{
Fan LEI $^{1}$, Guiwu WEI ${ }^{1,2}$, Weijie SHEN ${ }^{3}$, Yanfeng GUO ${ }^{*}$ \\ ${ }^{1}$ School of Mathematical Sciences, Sichuan Normal University, 610066 Chengdu, P.R. China \\ ${ }^{2}$ School of Business, Sichuan Normal University, 610101 Chengdu, P.R. China \\ ${ }^{3}$ School of Economics and Management, Chongqing University of Arts and Sciences, \\ 402160 Chongqing, P. R. China \\ ${ }^{4}$ School of Finance, Southwestern University of Finance and Economics, 610074 Chengdu, China
}

Received 18 January 2021; accepted 05 October 2021; first published online 15 December 2021

\begin{abstract}
With the rapid development of 3D printing technology, 3D printers are manufactured based on the principle of 3D printing technology are more and more widely used in the manufacturing industry. Choosing high quality $3 \mathrm{D}$ printers for industrial production is of great significance to the economic growth of enterprises. In fact, it is difficult to select the most optimal 3D printers under a single and simple standard. Therefore, this paper establishes the probabilistic double hierarchy linguistic EDAS (PDHL-EDAS) method for the multiple attribute group decision making (MAGDM). Then the CRITIC model is introduced to derive objective weight and the cumulative prospect theory is leaded into obtain the cumulative weight of PDHLTS. In addition, what's more, the PDHL-EDAS method is built and applied to the choice of high-quality 3D printer. Finally, compared with the available MAGDM methods under PDHLTS, the built method is proved to be scientific and effective.
\end{abstract}

Keywords: multiple attribute group decision making (MAGDM), probabilistic double hierarchy linguistic term set (PDHLTS), EDAS method, CRITIC method, 3D printer selection.

JEL Classification: C43, C61, D81.

\section{Introduction}

3D printing is a rapid prototyping information technology based on digital model files, which uses powder metal, plastic and other bonding materials to build objects by stacking and accumulating layer by layer (i.e. "layer by layer molding method"). In the past, it is often employed in mold manufacturing, industrial design and other fields to make models, but now it is gradually used directly to manufacture some products. In particular, it is used for the

\footnotetext{
*Corresponding author. E-mail: guoyanfeng@swufe.edu.cn
} 
production of some high-value products (such as animal hip joints or teeth, or some parts of automobiles and aircraft). At present, there are many researches on the improvement of 3D printing information technology and the application of 3D printer (Cheng et al., 2020; Chrispin et al., 2020; Liu et al., 2019; Pavan et al., 2021; Yi et al., 2019), but there are few researches on the selection and evaluation of 3D printer. Such as, Prabhu and Ilangkumaran (2019a) used VIKOR method to rank and evaluate 3D printers in fuzzy environment; Prabhu and Ilangkumaran (2019b) combined the GRA Method with TOPSIS method, and put forward the GRA-TOPSIS method to rank 3D printers in real environment.

Through the above literature, we can know that the research on $3 \mathrm{D}$ printer evaluation model is very few, and the evaluation information is given in the form of real number. But in the actual production and life, decision makers are more accustomed to using linguistic to describe the evaluation information. Pang, Wang, and Xu (2016) built the probabilistic linguistic term sets (PLTSs). Wei, Lin, Lu, Wu, and Wei (2021b) built the generalized dice similarity measures for PUL-MAGDM. Wei, Wu, Guo, and Wei (2021a) built the CODAS in probabilistic uncertain linguistic setting. But some complex linguistic evaluation information can't describe. for example, the frequency or probabilistic of "just right poor" is 0.3 , and "only a little good" is 0.7 is can not represent by those sets. Recently, to solve this issue the probabilistic double hierarchy linguistic term set (PDHLTS) proposed by Gou, Xu, Liao, and Herrera (2021). and Lei, Wei, and Chen (2021) defined the probabilistic double hierarchy linguistic CODAS method. So in PDHLTS, we can use $\left\{z_{0<h_{-1}>}(0.3), z_{1<h_{-2}>}(0.7)\right\}$ to represent The frequency or probabilistic of "just right poor" is 0.3 , and "only a little good" is 0.7 , where, $z=\left\{z_{-3}=\right.$ extremely poor, $z_{-2}=$ very poor, $z_{-1}=$ poor, $z_{0}=$ medium, $z_{1}=$ good, $z_{2}=$ very good, $z_{3}=$ extremely good $\}$,

$h=\left\{h_{-3}=\right.$ far form, $h_{-2}=$ only a little, $h_{-1}=$ a little, $h_{0}=j u s t$ right , $h_{1}=$ much, $h_{2}=$ very much, $h_{3}=$ extirely $\}$.

Besides, in order to better select the $3 \mathrm{D}$ printers that meet the actual production needs of enterprises, we will use EDAS model to evaluate the 3D printers. The EDAS is developed by Ghorabaee, Zavadskas, Olfat, and Turskis (2015), which is a novel and efficient method to solve MAGDM problems. Karunanithi et al. (2015) expanded the EDAS method to evaluate treatment outcome in Moyamoya disease. Stanujkic, Zavadskas, Keshavarz Ghorabaee, and Turskis (2017) proposed the EDAS model under interval grey numbers. Gundogdu, Kahraman, and Civan (2018) defined the HF-EDAS for hospital selection. Liang, Zhao, and Luo (2018) proposed that the cleanliness of gold production can be evaluated by a combination method of the EDAs method and ELECTRE model. Feng, Wei, and Liu (2018) extended hesitant fuzzy linguistic EDAS model to solve a company's investment issue. Kundakci (2019) developed the MACBETH and EDAS for selecting the steam boiler. Wang, Wang, and Wei (2019) employed EDAS to cope with the 2-tuple linguistic neutrosophic MADM. Darko and Liang (2020) combined EDAS method with Hamacher operators to select mobile payment platform. Wei, Wei, and Guo (2021c) defined the EDAS method for PL-MAGDM. He et al. (2019) built the EDAS algorithm for MAGDM with PULTSs.

From the above description, we can know that EDAS method is an efficient method to deal with MAGDM issue, but there is no research on EDAS method under PDHLTSs in the 
existing literatures, therefore, in order to effectively solve the problem of 3D printer selection, this paper will construct an PDHL-EDAS method to deal with this problem. The main work of this paper could be showed: (1) the PDHL-EDAS method for MAGDM is established; (2) the CRITIC (Diakoulaki et al., 1995) method is used to calculate the objective weight of attributes under PDHLTSs; (3) the steps of solving MAGDM problem with PDHL-EDAS model are given; (4) the PDHL-EDAS model is used to select the best 3D printer; (5) the model constructed in this paper is compared with the existing models.

\section{Preliminaries}

The notion of PDHLTS is reviewed in this section.

Definition 1 (Gouetal.,2017). Let $D H L=\left\{z_{\phi<h_{\psi}}>\mid \phi=-\chi, \cdots,-1,0,1, \cdots \chi ; \psi=-\eta, \cdots,-1,0,1, \cdots \eta\right\}$ be a DHLTS, the definition of the DHHFLTS is built as follows:

$$
D H H F L=\left\{z_{\phi<h_{\psi}>}^{k} \mid \phi=-\chi, \cdots,-1,0,1, \cdots \chi ; \psi=-\eta, \cdots,-1,0,1, \cdots \eta\right\},
$$

where $k=1,2, \ldots, \# D H L$, the $k$-th double hierarchy linguistic element (DHLE) is denoted as $z_{\phi<h_{\psi}>}^{k}$, the numbers of all DHLEs in DHL is expressed as \#DHL, and each DHLE in a DHHFLTS is arranged in ascending order.

Definition 2(Gouetal.,2017). Let $D H L=\left\{z_{\phi<h_{\psi}>} \mid \phi=-\chi, \cdots,-1,0,1, \cdots \chi ; \psi=-\eta, \cdots,-1,0,1, \cdots \eta\right\}$ be a DHLTS, the PDHLTS is built as follows:

$$
\operatorname{PDHL}(p)=\left\{z_{\phi<h_{\psi}>}^{k}\left(p^{k}\right) \mid z_{\phi<h_{\psi}>}^{k} \in D H L, p^{k} \geq 0, \quad \sum_{k=1}^{\# P H L(P)} p^{k} \leq 1\right\},
$$

where $k=1,2, \ldots, \# \operatorname{PDHL}(P) ; \phi=-\chi, \cdots,-1,0,1, \cdots \chi ; \psi=-\eta, \cdots,-1,0,1, \cdots \eta$, the $k$-th probabilistic double hierarchy linguistic element (PDHLE) is denoted as $z_{\phi<h_{\psi}>}^{k}\left(p^{k}\right)$, the numbers of all PDHLEs in PDHL(P) are expressed as \#PDHL(P), and According to $f\left(l_{\varphi<S_{\psi}>}^{k}\right)$, each PDHLE in a PDHLTS is arranged in ascending order, where the transformation function $f$ is defined by Eq. (3).

Definition 3(Gouetal.,2021). Let $D H L=\left\{z_{\phi<h_{\psi}>} \mid \phi=-\chi, \cdots,-1,0,1, \cdots \chi ; \psi=-\eta, \cdots,-1,0,1, \cdots \eta\right\}$ be a DHLTS, and $\operatorname{PDHL}(p)=\left\{z_{\phi<h_{\psi}>}^{k}\left(p^{k}\right) \mid z_{\phi<h_{\psi}>}^{k} \in D H L, p^{k} \geq 0, \sum_{k=1}^{\# P D H L(P)} p^{k} \leq 1\right\}$ be a PDHLTS, the equivalent information between the subscript $(\varphi, \psi)$ of $z_{\phi<h_{\psi}}^{k}>$ and the numerical $\gamma_{n}$ is converted by transformation functions $f$ and $f^{-1}$ :

$$
\begin{aligned}
& f:[-\chi, \chi] \times[-\eta, \eta] \rightarrow[0,1], f(\phi, \psi)=\frac{\psi+(\chi+\phi) \eta}{2 \chi \eta}=\gamma_{n} ; \\
& f^{-1}:[0,1] \rightarrow[-\chi, \chi] \times[-\eta, \eta], \\
& f^{-1}\left(\chi_{n}\right)=\left[2 \chi \gamma_{n}-\chi\right]_{<h_{\eta\left(\left(2 \chi \gamma_{n}-\chi\right)-\left[2 \chi \gamma_{n}-\chi\right]\right)}>\text { or }}\left[2 \chi \gamma_{n}-\chi\right]+1_{<h_{\eta\left(\left(2 \chi \gamma_{n}-\chi\right)-\left[2 \chi \gamma_{n}-\chi\right]\right)-\eta}}{ }^{*}
\end{aligned}
$$

Considering that the probability sum of all PDHLEs in PDHLTS may not be 1 , the following normalization formula is proposed. 


$$
\operatorname{PDHL}(p)=\left\{z_{\phi<h_{\psi}>}^{k}\left(p^{k}\right) \mid z_{\phi<h_{\psi}>}^{k} \in D H L, p^{k} \geq 0, \quad \sum_{k=1}^{\# P D H L(P)} p^{k}<1\right\},
$$

where $\tilde{p}^{k}=p^{k} / \sum_{k=1}^{\# P D H L(P)} p^{k} ; \phi \in[-\chi, \chi] ; \psi \in[-\eta, \eta] ; \varphi, \psi$ are integer numbers.

Definition 4 (Lei et al., 2021). Let $D H L=\left\{z_{\phi<h_{\psi}>} \mid \phi=-\chi, \cdots,-1,0,1, \cdots \chi ; \psi=-\eta, \cdots,-1,0,1, \cdots \eta\right\}$ be a DHLTS, $P D H \tilde{L}_{1}(\tilde{p})=\left\{z_{1 \phi<h_{\psi}>}^{k}\left(\tilde{p}_{1}^{k}\right) \mid z_{1 \phi<h_{\psi}>}^{k} \in D H L ; k=1,2, \ldots, \# P D H \tilde{L}_{1}(\tilde{p})\right\}$ and $\operatorname{PDH}_{2}(\tilde{p})=\left\{z_{2 \phi<h_{\psi}>}^{k}\left(\tilde{p}_{2}^{k}\right) \mid z_{2 \phi<h_{\psi}>}^{k} \in D H L ; k=1,2, \ldots, \# P D H \tilde{L}_{2}(\tilde{p})\right\}$ be two PDHLTSs, where

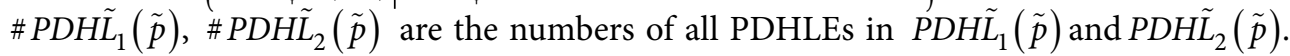
Especially, if $\# P D H \tilde{L}_{1}(\tilde{p})>\# P D H \tilde{L}_{2}(\tilde{p})$, then the numbers of \#PDH$\tilde{L}_{1}(\tilde{p})-\# P D H \tilde{L}_{2}(\tilde{p})$ DHLEs are added to $P D H \tilde{L}_{2}(\tilde{p})$. What's more, the newly added DHLEs need be the smallest DHLEs in $\mathrm{PDH}_{2}(\tilde{p})$ and the corresponding probabilities of newly added DHLTS should be zero.

Definition 5 (Lei et al., 2021). Let $P D H \tilde{L}(\tilde{p})=\left\{z_{\phi<h_{\psi}>}^{k}\left(\tilde{p}^{k}\right) \mid z_{\phi<h_{\psi}>}^{k} \in D H \tilde{L} ; k=1,2, \ldots\right.$, $\# P D H \tilde{L}(\tilde{p})\}$ be a PDHLTS, established the expected values $E(P D H \tilde{L}(\tilde{p}))$ and deviation degree $\sigma(P D H \tilde{L}(\tilde{p}))$ of $P D H \tilde{L}(\tilde{p})$ as follows:

$$
\begin{aligned}
& E(\operatorname{PDH} \tilde{L}(\tilde{p}))=\sum_{k=1}^{\# P D H \tilde{L}(\tilde{p})} f(\operatorname{PDH} \tilde{L}(\tilde{p})) \tilde{p}^{k} / \sum_{k=1}^{\# P D H \tilde{L}(\tilde{p})} \tilde{p}^{k} ; \\
& \sigma(\operatorname{PDH} \tilde{L}(\tilde{p}))=\sqrt{\sum_{k=1}^{\# P H \tilde{L}(\tilde{p})}\left(f(\operatorname{PDH} \tilde{L}(\tilde{p})) \tilde{p}^{k}-E(P D H \tilde{L}(\tilde{p}))\right)^{2}} / \sum_{k=1}^{\# P D H \tilde{L}(\tilde{p})} \tilde{p}^{k} .
\end{aligned}
$$

The order relationship between the two PDHLTSs can be further determined by Eqs (6)-(7).

(1) if $E\left(P D H \tilde{L}_{1}(\tilde{p})\right)>E\left(P D H \tilde{L}_{2}(\tilde{p})\right)$, then $P D H \tilde{L}_{1}(\tilde{p})>P D H \tilde{L}_{2}(\tilde{p})$;

(2) if $E\left(P D H \tilde{L}_{1}(\tilde{p})\right)=E\left(P D H \tilde{L}_{2}(\tilde{p})\right)$, then if $\sigma\left(P D H \tilde{L}_{1}(\tilde{p})\right)=\sigma\left(P D H \tilde{L}_{2}(\tilde{p})\right)$, then $P D H \tilde{L}_{1}(\tilde{p})=P D H \tilde{L}_{2}(\tilde{p})$; then if $\sigma\left(P D H \tilde{L}_{1}(\tilde{p})\right)<\sigma\left(P D H \tilde{L}_{2}(\tilde{p})\right)$, then, $P D H \tilde{L}_{1}(\tilde{p})>P D H \tilde{L}_{2}(\tilde{p})$.

Definition 6 (Lei et al., 2021). Let $D H L=\left\{z_{\phi<h_{\psi}} \mid \phi=-\chi, \cdots,-1,0,1, \cdots \chi ; \psi=-\eta, \cdots,-1,0,1, \cdots \eta\right\}$ be a DHLTS, $P D H \tilde{L}_{1}(\tilde{p})=\left\{z_{1 \phi<h_{\psi}>}^{k}\left(\tilde{p}_{1}^{k}\right) \mid z_{1 \phi<h_{\psi}>}^{k} \in D H L ; k=1,2, \ldots, \# P D H \tilde{L}_{1}(\tilde{p})\right\}$ and $P D H \tilde{L}_{2}(\tilde{p})=\left\{z_{2 \phi<h_{\psi}>}^{k}\left(\tilde{p}_{2}^{k}\right) \mid z_{2 \phi<h_{\psi}>}^{k} \in D H L ; k=1,2, \ldots, \# P D H \tilde{L}_{2}(\tilde{p})\right\}$ are two PDHLTSs, where \#PDH $\tilde{L}_{1}(\tilde{p})=\# P D H \tilde{L}_{2}(\tilde{p})=\# P D H \tilde{L}(\tilde{p})$, then Euclidean distance $E D\left(P D H \tilde{L}_{1}(\tilde{p})\right.$, $\left.P D H \tilde{L}_{2}(\tilde{p})\right)$ and Hamming distance $H D\left(P D H \tilde{L}_{1}(\tilde{p}), P D H \tilde{L}_{2}(\tilde{p})\right)$ between $P D H \tilde{L}_{1}(\tilde{p})$ and $\operatorname{PDH}_{2}(\tilde{p})$ are established.

$$
E D\left(P D H \tilde{L}_{1}(\tilde{p}), P D H \tilde{L}_{2}(\tilde{p})\right)=\sqrt{\frac{\sum_{k=1}^{\# P H \tilde{L}(\tilde{p})}\left(f\left(z_{1 \phi<h_{\psi}>}^{k}\right) \tilde{p}_{1}^{k}-f\left(z_{2 \phi<h_{\psi}>}^{k}\right) \tilde{p}_{2}^{k}\right)^{2}}{\# P D H \tilde{L}(\tilde{p})}} ;
$$




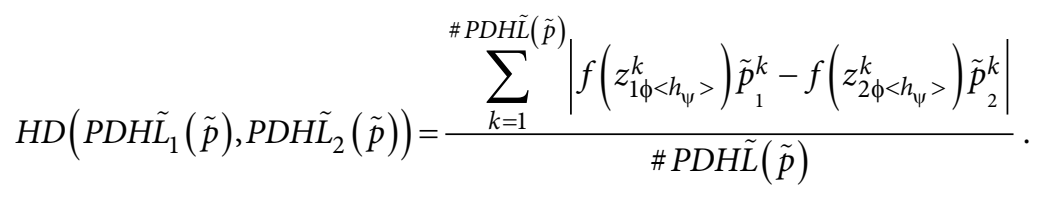

In order to facilitate readers to understand these two distance formulas, the following example is built.

Let $D H L=\left\{z_{\phi<h_{\psi}>} \mid \phi=-3, \cdots,-1,0,1, \cdots 3 ; \psi=-3, \cdots,-1,0,1, \cdots 3\right\}$ is a DHLTS, and $P D H \tilde{L}_{1}(\tilde{p})=\left\{z_{0<h_{1}>}(0.3), z_{0<h_{2}>}(0.4), z_{2<h_{1}>}(0.3)\right\}, P D H \tilde{L}_{2}(\tilde{p})=\left\{z_{1<h_{2}>}(0.5), z_{2<h_{0}>}(0.2), z_{2<h_{2}>}(0.3)\right\}$ are two PDLTSs, the Euclidean distance $E D\left(P D H \tilde{L}_{1}(\tilde{p}), P D H \tilde{L}_{2}(\tilde{p})\right)$ and Hamming distance $H D\left(P D H \tilde{L}_{1}(\tilde{p}), P D H \tilde{L}_{2}(\tilde{p})\right)$ can be obtained as follows:

According to Eq. (3)

$$
\begin{aligned}
& f\left(z_{0<h_{1}>}\right)=\frac{1+(3+0) \times 3}{2 \times 3 \times 3}=\frac{10}{18}, f\left(z_{0<h_{2}>}\right)=\frac{2+(3+0) \times 3}{2 \times 3 \times 3}=\frac{11}{18}, f\left(z_{2<h_{1}>}\right)=\frac{1+(3+2) \times 3}{2 \times 3 \times 3}=\frac{16}{18}, \\
& f\left(z_{1<h_{2}>}\right)=\frac{2+(3+1) \times 3}{2 \times 3 \times 3}=\frac{14}{18}, f\left(z_{2<h_{0}>}\right)=\frac{0+(3+2) \times 3}{2 \times 3 \times 3}=\frac{15}{18}, f\left(z_{2<h_{2}>}\right)=\frac{2+(3+2) \times 3}{2 \times 3 \times 3}=\frac{17}{18} .
\end{aligned}
$$

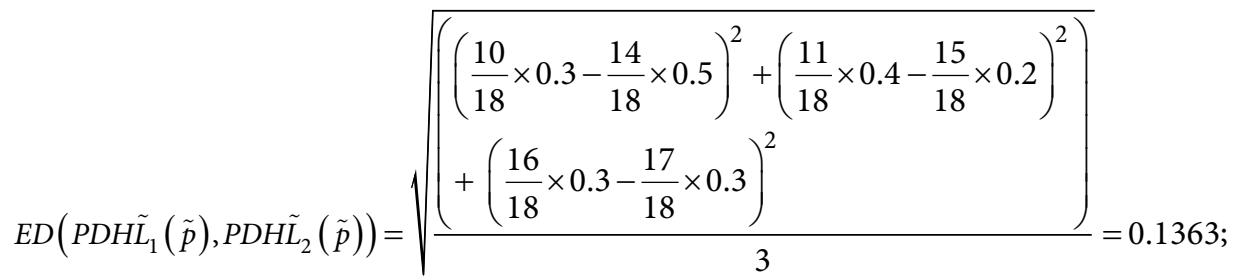

$$
H D\left(P D H \tilde{L}_{1}(\tilde{p}), P D H \tilde{L}_{2}(\tilde{p})\right)=\frac{\left(\begin{array}{l}
\left|\frac{10}{18} \times 0.3-\frac{14}{18} \times 0.5\right|+\left|\frac{11}{18} \times 0.4-\frac{15}{18} \times 0.2\right| \\
+\left|\frac{16}{18} \times 0.3-\frac{17}{18} \times 0.3\right|
\end{array}\right)}{3}=0.1055
$$

\section{PDHL-EDAS method for MAGDM with combined weight}

Now, EDAS method for PDHLTSs is proposed to tackle MAGDM problems. And the MAGDM issues are represented by the following mathematical symbols. All alternatives is denoted as $A L=\left\{A L_{1}, A L_{2}, \cdots, A L_{m}\right\}$, the $A T=\left\{A T_{1}, A T_{2}, \cdots, A T_{n}\right\}$ is denoted the set of attributes, and combined weight vector is $c w=\left(c w_{1}, c w_{2}, \cdots, c w_{n}\right)$, where $c w_{j} \in[0,1], j=1,2, \cdots, n, \sum_{j=1}^{n} c w_{j}=1$, and $E=\left\{E X_{1}, E X_{2}, \cdots, E X_{g}\right\}$ be a set of experts. Suppose that $\mathrm{t}$-th expert $E X_{t}$ is evaluated $\mathrm{i}$-th alternative $C A_{i}$ under $\mathrm{j}$-th attribute $A T_{j}$ as $\operatorname{PDHL}_{i j}{ }^{(t)}(p)=\left\{z_{i j \phi<h_{\psi}>}^{k}{ }^{(t)}\left(p_{i j}{ }^{k(t)}\right) \mid z_{i j \phi<h_{\psi}>}^{k}{ }^{(t)} \in D H L, p_{i j}{ }^{k(t)} \geq 0, \quad \sum_{k=1}^{\# P D H(P)} p_{i j}{ }^{k(t)} \leq 1\right\}$, $(i=1,2, \cdots, m, j=1,2, \cdots, n, t=1,2, \cdots, g)$. 
Then, PDHL-EDAS method is built to tackle MAGDM issue with combined attribute weight.

Step 1. Established all decision makers' decision matrixes PDHLTS $Q^{(t)}=\left(P D H L^{(t)}(P)\right)_{m \times n}$.

Step 2. Converted cost index into benefit index. LetPDH $\tilde{L}(\tilde{p})=\left\{z_{\phi<h_{\psi}>}^{k}\left(\tilde{p}^{k}\right) \mid z_{\phi<h_{\psi}>}^{k} \in\right.$ $D H \tilde{L} ; k=1,2, \ldots, P D H \tilde{L}(\tilde{p})\}$ be a PDHLTS, if $z_{\phi<h_{\psi}>}^{k}\left(\tilde{p}^{k}\right)$ is a cost linguistic evaluation, convert it into the corresponding benefit evaluation $z_{-\phi<-h_{\psi}>}^{k}\left(\tilde{p}^{k}\right)$.

Step 3. computed the normalized decision matrix $\tilde{Q}^{(t)}=\left(P D H \tilde{L}_{i j}(t)(\tilde{p})\right)_{m \times n}$.

Step 4. Calculated the PDHLTS prospect weight of each alternative, and obtain The PDHLTSs decision making information with prospects weight of each alternative based on each expert.

Firstly, the probabilistic double hierarchy linguistic prospect values $P W_{i j}^{(t)}(\tilde{p})$ of each alternative is obtained.

$$
\begin{aligned}
& \text { if: } \frac{\sum_{k=1}^{\# P D H \tilde{L}(\tilde{p})} f\left(z_{i j \phi<h_{\psi}>}^{k}{ }^{(t)}\right) \tilde{p}_{i j}^{k(t)}-f\left(\overline{z_{j \phi<h_{\psi}>}^{k}(t)}\right) \overline{p_{j}^{k(t)}}}{\# P D H \tilde{L}(\tilde{p})} \geq 0, \\
& \text { then, } P W_{i j}^{(t)}(\tilde{p})=\left(H D\left(P D H \tilde{L}_{i j}^{(t)}(\tilde{p}), \overline{P D H \tilde{L}_{j}^{(t)}}\left(\overline{\tilde{p}_{j}^{(t)}}\right)\right)\right)^{\alpha} ; \\
& \text { if: } \frac{\sum_{k=1}^{\# P H \tilde{L}(\tilde{p})} f\left(z_{i j \phi<h_{\psi}>}^{k}(t)\right) \tilde{p}_{i j}^{k(t)}-f\left(\overline{z_{j \phi<h_{\psi}>}^{k}(t)}\right) \overline{p_{j}^{k(t)}}}{\# P D H \tilde{L}(\tilde{p})}<0, \\
& \text { then, } P W_{i j}^{(t)}(\tilde{p})=-\lambda\left(H D\left(P D H \tilde{L}_{i j}^{(t)}(\tilde{p}), \overline{P D H \tilde{L}_{j}^{(t)}}\left(\overline{\tilde{p}_{j}^{(t)}}\right)\right)\right)^{\beta},
\end{aligned}
$$

where

$$
\begin{aligned}
& H D\left(P D H \tilde{L}_{i j}{ }^{(t)}(\tilde{p}), \overline{P D H \tilde{L}}_{j}^{(t)}(\tilde{p})\right)=\frac{\sum_{k=1}^{\# P D H \tilde{L}}(\tilde{p})\left|f\left(z_{i j \phi<h_{\psi}>}^{k}{ }^{(t)}\right) \tilde{p}_{i j}^{k(t)}-f\left(\overline{z_{j \phi<h_{\psi}>}^{k}{ }^{(t)}}\right) \overline{p_{j}^{k(t)}}\right|}{\# P D H \tilde{L}(\tilde{p})}, \\
& \text { particularly } \overline{P D H \tilde{L}_{j}^{(t)}}\left(\overline{\tilde{p}_{j}^{(t)}}\right)=\left\{\overline{z_{j \phi h_{\psi}>}^{k}(t)}\left(\overline{p_{j}^{k(t)}}\right) \mid k=1,2, \ldots, P D H \tilde{L}^{(t)}\left(\tilde{p}^{(t)}\right)\right\} \text {; }
\end{aligned}
$$

$$
\left\{\overline{z_{j \phi<h_{\psi}>}^{k}(t)}\left(\overline{p_{j}^{k(t)}}\right)\right\}=\left(\frac{f^{-1}\left(\sum_{i=1}^{m} f\left(z_{i j \phi<h_{\psi}>}^{k}\right)\right)}{m}\left(\frac{\left.\sum_{i=1}^{m} \tilde{p}_{i j}^{k(t)}\right)}{m}\right)\right),
$$

with $\alpha=\beta=0.88, \lambda=2.25$.

Then, the normalized probabilistic double hierarchy linguistic prospect weights $N P W_{i j}{ }^{(t)}(\tilde{p})$ are calculated by Eqs (15)-(16):

$$
\omega_{i j}{ }^{(t)}=\frac{N P W_{i j}{ }^{(t)}(\tilde{p})}{\sum_{i=1}^{m} N P W_{i j}{ }^{(t)}(\tilde{p})},
$$


where $N P W_{i j}^{(t)}(\tilde{p})=\frac{P W_{i j}^{(t)}(\tilde{p})-(-2.25)}{1-(-2.25)}, \omega_{i j}^{(t)} \in[0,1], \sum_{i=1}^{m} \omega_{i j}{ }^{(t)}=1$.

In addition, we can obtain The PDHLTSs decision matrix $P W \tilde{Q}^{(t)}=\left(P W P D H \tilde{L}_{i j}{ }^{(t)}(\tilde{p})\right)_{m \times n}$ with prospects weight of each alternative based on each expert by Eq. (17). And we can obtain the overall prospect weight PDHLTSs decision matrix $P W \tilde{Q}=\left(P W P D H \tilde{L}_{i j}(\tilde{p})\right)_{m \times n}$. $\left(P W P D H \tilde{L}_{i j}{ }^{(t)}(\tilde{p})\right)_{m \times n}=\omega_{i j}{ }^{(t)}\left(P D H \tilde{L}_{i j}{ }^{(t)}(\tilde{p})\right)=\left(f^{-1}\left(\sum_{k=1}^{\# P D H \tilde{L}_{i j}(t)} \omega_{i j}^{(t)} f\left(z_{i j \phi<h_{\psi}>}{ }^{k}(t)\right)\right), \omega_{i j}{ }^{(t)} \sum_{k=1}^{\# P D H \tilde{L}_{i j}^{(t)}} \tilde{p}_{i j}^{k(t)}\right)$.

Finally, we can calculate the normalized overall prospect weight PDHLTSs decision ma$\operatorname{trix} N P W \tilde{Q}=\left(N P W P D H \tilde{L}_{i j}(\tilde{p})\right)_{m \times n}$.

Step 5. Calculated the combined weight.

The CRITIC method is proposed by Diakoulaki et al. (1995), which is a comprehensive measure of the objective weight of attributes. Now, the CRITIC method is extended in PDHLTSs to calculate the objective weight of attributes, and detailed calculation steps are as follows:

(1) Calculated between attributes' coefficient of correlation to establish the probabilistic double hierarchy linguistic coefficient of correlation matrix PWPDHLCCM= $\left(P W P D H L C C_{j r}\right)_{n \times n}$ as follows:

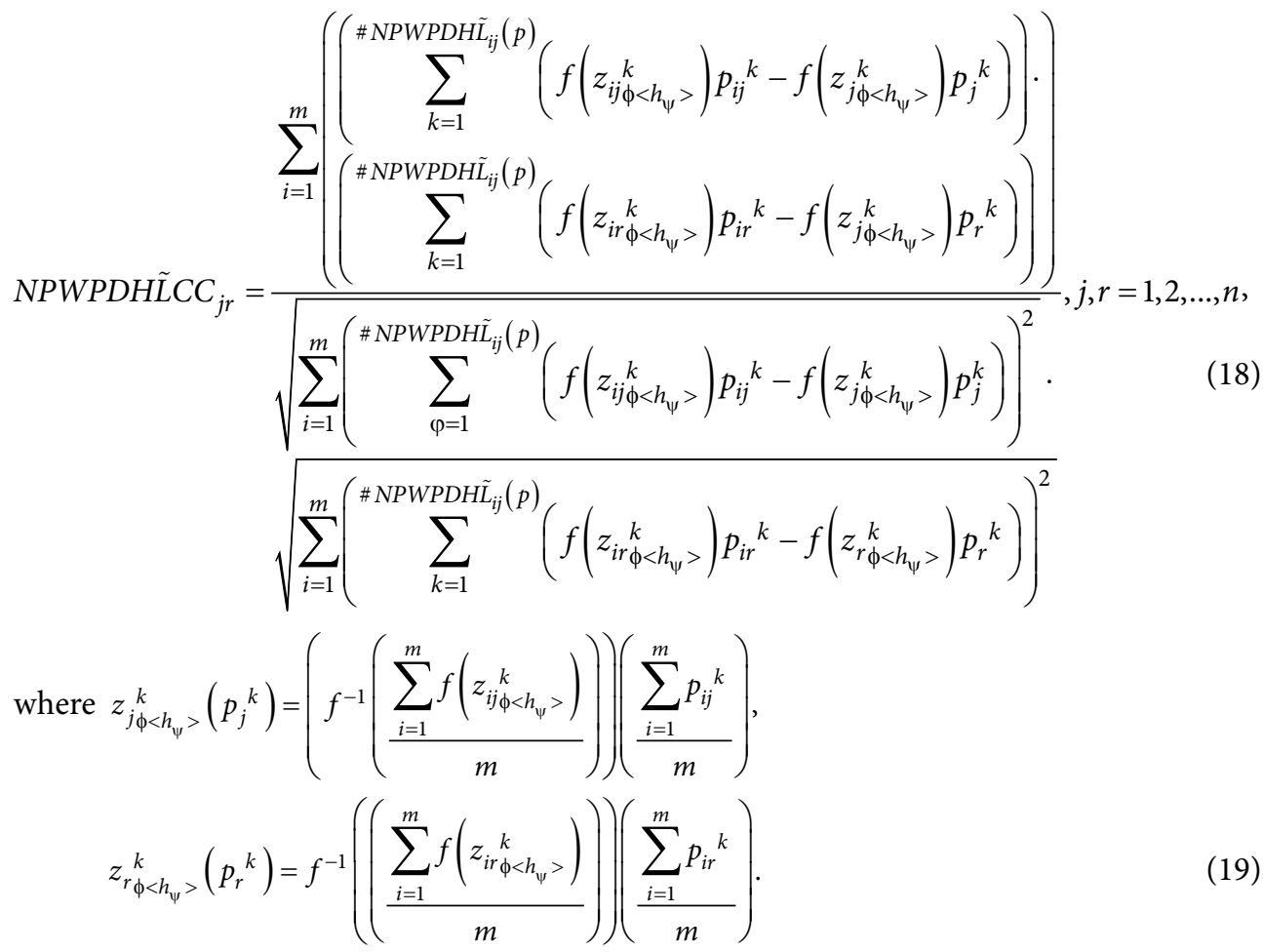


(2) Probabilistic double hierarchy linguistic standard deviation (NPWPDHLSD) of each attribute could be got as follows:

$$
N P W P D H \tilde{L} S D_{j}=\sqrt{\frac{1}{m-1} \sum_{i=1}^{m}\left(\sum_{k=1}^{\# N P W P D H \tilde{L}_{i j}(p)}\left(f\left(z_{i j \phi<h_{\psi}>}^{k}\right) p_{i j}^{k}-f\left(z_{j \phi<h_{\psi}>}^{k}\right) p_{j}^{k}\right)\right)^{2}},
$$

(3) The objective weights of all attributes can be built as follows:

$$
o w_{j}=\frac{N P W P D H \tilde{L} S D_{j} \sum_{r=1}^{n}\left(1-N P W P D H \tilde{L} C C_{j r}\right)}{\sum_{j=1}^{n}\left(N P W P D H \tilde{L} S D_{j} \sum_{r=1}^{n}\left(1-N P W P D H \tilde{L} C C_{j r}\right)\right)}, j=1,2 \ldots, n,
$$

where $o \omega_{j} \in[0,1], \sum_{j=1}^{n} o \omega_{j}=1$.

Then, assumed $s \omega_{j} \in[0,1], \sum_{j=1}^{n} s \omega_{j}=1, j=1,2, \ldots, n$ is the subjective weight provided by expert. Therefore, the comprehensive weight is built:

where $c \omega_{j} \in[0,1], \sum_{j=1}^{n} c \omega_{j}=1, j=1,2, \ldots, n$.

$$
c \omega_{j}=\frac{\sqrt{o \omega_{j} s \omega_{j}}}{\sum_{j=1}^{n} \sqrt{o \omega_{j} s \omega_{j}}}
$$

Step 6. The probabilistic double hierarchy linguistic average values are obtained by Eqs (23)-(25):

$$
\begin{aligned}
& \operatorname{NPWAVPDH\tilde {L}}(\tilde{p})=\left(\operatorname{NPWAVPDH} \tilde{L}_{j}(\tilde{p})\right)_{1 \times n} ; \\
& N P W A V P D H \tilde{L}_{j}(\tilde{p})=\left\{z_{j \phi<h_{\psi}>}^{k}\left(p_{j}^{k}\right) \mid k=1,2, \ldots, \# N P W A P D H \tilde{L}(\tilde{p})\right\} ; \\
& z_{j \phi<h_{\psi}>}^{k}\left(p_{j}{ }^{k}\right)=\left\{\frac{f^{-1}\left(\sum_{i=1}^{m} f\left(z_{i j \phi<h_{\psi}>}^{k}(t)\right)\right.}{m},\left(\frac{\left.\sum_{i=1}^{m} \tilde{p}_{i j}{ }^{k(t)}\right)}{m}\right)\right\} .
\end{aligned}
$$

Step 7. The positive distance of probabilistic double hierarchy linguistic from average (NPWPDHLPDA) can be calculated by Eq. (26), and the positive distance of probabilistic double hierarchy linguistic from average (NPWPDHLNDA) can be obtained by Eq. (27):

$$
\begin{aligned}
& \operatorname{NPWPDH} \tilde{L}^{P D A}(\tilde{p})=\left[N P W P D H \tilde{L}_{i j}{ }^{P D A}(\tilde{p})\right]_{m \times n} ; \\
& N P W P D H \tilde{L}^{N D A}(\tilde{p})=\left[N P W P D H \tilde{L}_{i j}{ }^{N D A}(\tilde{p})\right]_{m \times n} .
\end{aligned}
$$

Respectively, beneficial attributes are computed by Eqs (28)-(29): 


$$
\begin{aligned}
& N P W P D H \tilde{L}_{i j}{ }^{P D A}(\tilde{p})=\frac{\max \left(d\left(N P W P D H \tilde{L}_{i j}(\tilde{p}), N P W A V P D H \tilde{L}_{j}(\tilde{p})\right), 0\right)}{E\left(N P W A V P D H \tilde{L}_{j}(\tilde{p})\right)} ; \\
& \operatorname{NPWPDH} \tilde{L}_{i j}{ }^{N D A}(\tilde{p})=\frac{\max \left(d\left(N P W A V P D H \tilde{L}_{j}(\tilde{p}), N P W P D H \tilde{L}_{i j}(\tilde{p})\right), 0\right)}{E\left(N P W A V P D H \tilde{L}_{j}(\tilde{p})\right)} ;
\end{aligned}
$$

Then, cost attributes are computed by Eqs (30)-(31):

$$
\begin{aligned}
& N P W P D H \tilde{L}_{i j}{ }^{P D A}(\tilde{p})=\frac{\max \left(d\left(N P W A V P D H \tilde{L}_{j}(\tilde{p}), N P W P D H \tilde{L}_{i j}(\tilde{p})\right), 0\right)}{E\left(N P W A V P D H \tilde{L}_{j}(\tilde{p})\right)} ; \\
& N P W P D H \tilde{L}_{i j}^{N D A}(\tilde{p})=\frac{\max \left(d\left(N P W P D H \tilde{L}_{i j}(\tilde{p}), N P W A V P D H \tilde{L}_{j}(\tilde{p})\right), 0\right)}{E\left(N P W A V P D H \tilde{L}_{j}(\tilde{p})\right)} .
\end{aligned}
$$

Step 8. Computing the weight sum of NPWPDHLPDA and weight sum of NPWPDHLNDA for all alternatives as:

$$
\begin{aligned}
& N P W P D H \tilde{L}_{i}^{S P}(\tilde{p})=\sum_{j=1}^{n} c \omega_{j} N P W P D H \tilde{L}_{i j} P D A(\tilde{p}) ; \\
& N P W P D H \tilde{L}_{i}^{S N}(\tilde{p})=\sum_{j=1}^{n} c \omega_{j} N P W P D H \tilde{L}_{i j} N D A(\tilde{p}) .
\end{aligned}
$$

Step 9. The normalized values of $N P W P D H{ }^{j}{ }_{i} S P(\tilde{p})$ and $N P W P D H \tilde{L}_{i}{ }^{N P}(\tilde{p})$ can be obtained by Eqs (34)-(35):

$$
\begin{array}{rl}
N P W P D H \tilde{L}_{i} & N S P(\tilde{p})=\frac{N P W P D H \tilde{L}_{i}^{S P}(\tilde{p})}{\max _{i=1} N P W P D H \tilde{L}_{i}^{S P}(\tilde{p})} ; \\
N P W P D H \tilde{L}_{i}^{N S N}(\tilde{p}) & =1-\frac{N P W P D H \tilde{L}_{i}^{S N}(\tilde{p})}{\max _{i=1} N P W P D H \tilde{L}_{i}^{S N}(\tilde{p})} .
\end{array}
$$

Step 10. Computed the assessment value $N P W P D H \tilde{L}_{i}{ }^{A S}(\tilde{p})$ of every alternative:

$$
N P W P D H \tilde{L}_{i}^{A S}(\tilde{p})=\frac{N P W P D H \tilde{L}_{i}^{N S P}(\tilde{p})+N P W P D H \tilde{L}_{i}^{N S N}(\tilde{p})}{2} .
$$

Step 11. Used assessment value $N P W P D H \tilde{L}_{i}{ }^{A S}(\tilde{p})$ to rank the alternatives, and the larger $N P W P D H \tilde{L}_{i}{ }^{A S}(\tilde{p})$, the better the alternative is.

\section{Numerical example and comparative analysis}

\subsection{Numerical example}

With the continuous development of 3D printing technology, high-efficiency and lowcost 3D printers have been widely used in manufacturing. A high-end car manufacturer of a certain brand has researched and developed a new high-end car and plans to use a 3D printer to produce certain parts of the new car. Choosing high-quality $3 \mathrm{D}$ printer is closely 
related to the economic interests of the manufacturer. The high-quality $3 \mathrm{D}$ printer selection is also a classical MAGDM issue. Therefore, the PDHL-EDAS method is used to select the optimal 3D printer. Now, there are five 3D printers $D P=\left\{D P_{1}, D P_{2}, D P_{3}, D P_{4}, D P_{5}\right\}$ that meet the production conditions. Three invited experts $E=\left\{E X_{1}, E X_{2}, E X_{3}\right\}$ have selected four attributes $A T=\left\{A T_{1}, A T_{2}, A T_{3}, A T_{4}\right\}$ to evaluate the five given alternatives, And the $s \omega=[0.28,0.20,0.21,0.31]$ is the subjective weight given by the experts. In addition, $A T_{1}:$ Endurance of production materials. $A T_{2}$ : Cost of production. $A T_{3}$ : speed of Production $A T_{4}$ : After-sales service. Evidently, $A T_{2}$ is cost attribute, on the contrary, $A T_{1}, A T_{3}$ and $A T_{4}$ are benefit attributes, and $c w=\left(c w_{1}, c w_{2}, c w_{3}, c w_{4}\right)$ is the combined weight of four attributes where $c w_{j} \in[0,1], j=1,2,3,4, \sum_{j=1}^{4} c w_{j}=1$. Supposed that $t$-th expert $E X_{t}$ Evaluated $i$-th alternative $C A_{i}$ under $j$-th attribute $A T_{j}$ as

$\operatorname{PDHL}_{i j}{ }^{(t)}(p)=\left\{z_{i j \phi<h_{\psi}>}{ }^{k}{ }^{(t)}\left(p_{i j}{ }^{k(t)}\right) \mid z_{i j \phi<h_{\psi}>}^{k}{ }^{(t)} \in D H L, p_{i j}{ }^{k(t)} \geq 0, \quad \sum_{k=1}^{\# P D H(P)} p_{i j}{ }^{k(t)} \leq 1\right\}$, $(i=1,2, \cdots, 5, j=1,2, \cdots, 4, t=1,2,3)$ where, the double linguistic hierarchy evaluation information tables are given as follows:

$Z=\left\{z_{-3}=\right.$ extremely poor, $z_{-2}=$ very poor, $z_{-1}=$ poor,$z_{0}=$ medium,

$z_{1}=$ good,$z_{2}=$ very good, $z_{3}=$ extremely good $\}$,

$H=\left\{h_{-3}=\right.$ far form, $h_{-2}=$ only a little,$h_{-1}=$ a little,$h_{0}=$ just right,

$h_{1}=$ much $h_{2}=$ very much, $h_{2}=$ very much, $h_{3}=$ extirely $\}$.

Then, the decision matrixes of each invited expert are expressed in Tables 1-3.

Table 1. The PDHLTSs evaluation of all alternatives is provided by $\mathrm{e}_{1}$

\begin{tabular}{|l|l|l|}
\hline & \multicolumn{1}{|c|}{$\mathrm{AT}_{1}$} & \multicolumn{1}{|c|}{$\mathrm{AT}_{2}$} \\
\hline $\mathrm{DP}_{1}$ & $\left\{z_{-3<h_{0}>}(0.6), z_{-2<h_{1}>}(0.3), z_{-2<h_{2}>}(0.1)\right\}$ & $\left\{z_{1<h_{-2}>}(0.3), z_{2<h_{0}>}(0.5), z_{3<h_{-2}>}(0.2)\right\}$ \\
\hline $\mathrm{DP}_{2}$ & $\left\{z_{3<h_{-1}>}(0.0), z_{3<h_{-1}>}(0.1), z_{3<h_{0}>}(0.9)\right\}$ & $\left\{z_{-3<h_{0}>}(1.0)\right\}$ \\
\hline $\mathrm{DP}_{3}$ & $\left\{z_{0<h_{1}>}(0.3), z_{1<h_{0}>}(0.4), z_{2<h_{1}>}(0.3)\right\}$ & $\left\{z_{-3<h_{0}>}(0.2), z_{-2<h_{0}>}(0.3), z_{-1<h_{-2}>}(0.5)\right\}$ \\
\hline $\mathrm{DP}_{4}$ & $\left\{z_{-3<h_{2}>}(0.4), z_{-2<h_{1}>}(0.3), z_{-1<h_{2}>}(0.3)\right\}$ & $\left\{z_{1<h_{0}>}(0.3), z_{2<h_{-1}>}(0.2), z_{3<h_{0}>}(0.5)\right\}$ \\
\hline $\mathrm{DP}_{5}$ & $\left\{z_{-2<h_{0}>}(0.6), z_{-1<h_{0}>}(0.2), z_{0<h_{0}>}(0.2)\right\}$ & $\left\{z_{-2<h_{-1}>}(0.2), z_{-1<h_{-2}>}(0.5), z_{-1<h_{0}>}(0.3)\right\}$ \\
\hline
\end{tabular}

\begin{tabular}{|c|c|c|}
\hline & $\mathrm{AT}_{3}$ & $\mathrm{AT}_{4}$ \\
\hline $\mathrm{DP}_{1}$ & $\left\{z_{-2<h_{0}>}(0.3), z_{-2<h_{1}>}(0.3), z_{-1<h_{2}>}(0.4)\right\}$ & $\left\{z_{-3<h_{0}>}(0.1), z_{-3<h_{2}>}(0.5), z_{-2<h_{2}>}(0.4)\right\}$ \\
\hline $\mathrm{DP}_{2}$ & $\left\{z_{2<h_{0}>}(0.1), z_{3<h_{-1}>}(0.5), z_{3<h_{0}>}(0.4)\right\}$ & $\left\{z_{2<h_{0}>}(0.1), z_{3<h_{-1}>}(0.2), z_{3<h_{0}>}(0.7)\right\}$ \\
\hline $\mathrm{DP}_{3}$ & $\left\{z_{3<h_{-1}>}(0.0), z_{3<h_{-1}>}(0.6), z_{3<h_{0}>}(0.4)\right\}$ & $\left\{z_{1<h_{2}>}(0.7), z_{2<h_{0}>}(0.1), z_{3<h_{0}>}(0.2)\right\}$ \\
\hline
\end{tabular}


End of Table 1

\begin{tabular}{|c|c|c|}
\hline & $\mathrm{AT}_{3}$ & $\mathrm{AT}_{4}$ \\
\hline $\mathrm{DP}_{4}$ & $\left\{z_{-3<h_{0}>}(0.3), z_{-2<h_{1}>}(0.5), z_{-2<h_{2}>}(0.2)\right\}$ & $\left\{z_{-2<h_{1}>}(0.3), z_{-1<h_{1}>}(0.3), z_{0<h_{1}>}(0.4)\right\}$ \\
\hline $\mathrm{DP}_{5}$ & $\left\{z_{-1<h_{1}>}(0.5), z_{1<h_{0}>}(0.2), z_{2<h_{-2}>}(0.3)\right\}$ & $\left\{z_{3<h_{-2}>}(0.0), z_{3<h_{-2}>}(0.4), z_{3<h_{0}>}(0.6)\right\}$ \\
\hline
\end{tabular}

Table 2. The PDHLTSs evaluation of all alternatives is provided by $\mathrm{e}_{2}$

\begin{tabular}{|l|l|l|}
\hline & \multicolumn{1}{|c|}{$\mathrm{AT}_{1}$} & \multicolumn{1}{c|}{$\mathrm{AT}_{2}$} \\
\hline $\mathrm{DP}_{1}$ & $\left\{z_{-3<h_{0}>}(0.2), z_{-3<h_{2}>}(0.5), z_{-2<h_{1}>}(0.3)\right\}$ & $\left\{z_{2<h_{-1}>}(0.4), z_{3<h_{-2}>}(0.2), z_{3<h_{-1}>}(0.4)\right\}$ \\
\hline $\mathrm{DP}_{2}$ & $\left\{z_{3<h_{0}>}(0.0), z_{3<h_{0}>}(0.0), z_{3<h_{0}>}(1.0)\right\}$ & $\left\{l_{-3<s_{0}>}(0.8), l_{-3<s_{1}>}(0.1), l_{-3<s_{2}>}(0.1),\right\}$ \\
\hline $\mathrm{DP}_{3}$ & $\left\{z_{1<h_{1}>}(0.5), z_{2<h_{0}>}(0.3), z_{3<h_{-1}>}(0.2)\right\}$ & $\left\{z_{-3<h_{2}>}(0.4), z_{-2<h_{1}>}(0.2), z_{-2<h_{0}>}(0.4)\right\}$ \\
\hline $\mathrm{DP}_{4}$ & $\left\{z_{-3<h_{2}>}(0.3), z_{-2<h_{2}>}(0.4), z_{-1<h_{1}>}(0.3)\right\}$ & $\left\{z_{2<h_{-2}>}(0.2), z_{2<h_{-1}>}(0.2), z_{3<h_{-2}>}(0.6)\right\}$ \\
\hline $\mathrm{DP}_{5}$ & $\left\{z_{1<h_{0}>}(0.5), z_{1<h_{2}>}(0.3), z_{2<h_{1}>}(0.2)\right\}$ & $\left\{z_{-1<h_{-1}>}(0.2), z_{0<h_{-2}>}(0.1), z_{0<h_{-1}>}(0.7)\right\}$ \\
\hline
\end{tabular}

\begin{tabular}{|l|l|l|}
\hline & \multicolumn{1}{|c|}{$\mathrm{AT}_{3}$} & \multicolumn{1}{c|}{$\mathrm{AT}_{4}$} \\
\hline $\mathrm{DP}_{1}$ & $\left\{z_{-2<h_{1}>}(0.2), z_{-2<h_{2}>}(0.5), z_{-1<h_{2}>}(0.3)\right\}$ & $\left\{z_{-3<h_{0}>}(0.7), z_{-2<h_{0}>}(0.2), z_{-2<h_{1}>}(0.1)\right\}$ \\
\hline $\mathrm{DP}_{2}$ & $\left\{z_{3<h_{-1}>}(0.0), z_{3<h_{-1}>}(0.1), z_{3<h_{0}>}(0.9)\right\}$ & $\left\{z_{2<h_{0}>}(0.2), z_{3<h_{-2}>}(0.3), z_{3<h_{0}>}(0.5)\right\}$ \\
\hline $\mathrm{DP}_{3}$ & $\left\{z_{2<h_{0}>}(0.3), z_{3<h_{-2}>}(0.4), z_{3<h_{0}>}(0.3)\right\}$ & $\left\{z_{1<h_{1}>}(0.5), z_{2<h_{0}>}(0.3), z_{2<h_{1}>}(0.2)\right\}$ \\
\hline $\mathrm{DP}_{4}$ & $\left\{z_{-3<h_{1}>}(0.3), z_{-2<h_{0}>}(0.5), z_{-2<h_{1}>}(0.2)\right\}$ & $\left\{z_{-2<h_{1}>}(0.2), z_{-1<h_{0}>}(0.2), z_{-1<h_{2}>}(0.6)\right\}$ \\
\hline $\mathrm{DP}_{5}$ & $\left\{z_{0<h_{1}>}(0.7), z_{1<h_{0}>}(0.2), z_{1<h_{2}>}(0.1)\right\}$ & $\left\{z_{2<h_{0}>}(0.1), z_{3<h_{-1}>}(0.3), z_{3<h_{0}>}(0.6)\right\}$ \\
\hline
\end{tabular}

Table 3. The PDHLTSs evaluation of all alternatives is provided by $\mathrm{e}_{3}$

\begin{tabular}{|l|l|l|}
\hline & \multicolumn{1}{|c|}{$\mathrm{AT}_{1}$} & \multicolumn{1}{c|}{$\mathrm{AT}_{2}$} \\
\hline $\mathrm{DP}_{1}$ & $\left\{z_{-3<h_{2}>}(0.5), z_{-2<h_{0}>}(0.3), z_{-2<h_{1}>}(0.2)\right\}$ & $\left\{z_{2<h_{0}>}(0.2), z_{3<h_{-1}>}(0.4), z_{3<h_{0}>}(0.4)\right\}$ \\
\hline $\mathrm{DP}_{2}$ & $\left\{z_{3<h_{0}>}(1.0)\right\}$ & $\left\{z_{-3<h_{0}>}(0.8), z_{-3<h_{1}>}(0.2)\right\}$ \\
\hline $\mathrm{DP}_{3}$ & $\left\{z_{1<h_{2}>}(0.5), z_{2<h_{0}>}(0.3), z_{3<h_{-2}>}(0.2)\right\}$ & $\left\{z_{-3<h_{2}>}(0.5), z_{-2<h_{0}>}(0.1), z_{-2<h_{1}>}(0.4)\right\}$ \\
\hline $\mathrm{DP}_{4}$ & $\left\{z_{-3<h_{2}>}(0.1), z_{-2<h_{1}>}(0.6), z_{-1<h_{1}>}(0.3)\right\}$ & $\left\{z_{2<h_{-2}>}(0.1), z_{2<h_{-1}>}(0.2), z_{3<h_{-2}>}(0.7)\right\}$ \\
\hline $\mathrm{DP}_{5}$ & $\left\{z_{-1<h_{0}>}(0.4), z_{0<h_{2}>}(0.4), z_{2<h_{0}>}(0.2)\right\}$ & $\left\{z_{-2<h_{1}>}(0.1), z_{0<h_{-2}>}(0.3), z_{0<h_{-1}>}(0.6)\right\}$ \\
\hline
\end{tabular}


End of Table 3

\begin{tabular}{|l|l|l|}
\hline & \multicolumn{1}{|c|}{$\mathrm{AT}_{3}$} & \multicolumn{1}{c|}{$\mathrm{AT}_{4}$} \\
\hline $\mathrm{DP}_{1}$ & $\left\{z_{-3<h_{2}>}(0.2), z_{-2<h_{0}>}(0.3), z_{-1<h_{2}>}(0.5)\right\}$ & $\left\{z_{-3<h_{0}>}(0.4), z_{-3<h_{1}>}(0.6)\right\}$ \\
\hline $\mathrm{DP}_{2}$ & $\left\{z_{3<h_{-2}>}(0.2), z_{3<h_{-1}>}(0.4), z_{3<h_{0}>}(0.4)\right\}$ & $\left\{z_{3<h_{-2}>}(0.2), z_{3<h_{-1}>}(0.4), z_{3<h_{0}>}(0.4)\right\}$ \\
\hline $\mathrm{DP}_{3}$ & $\left\{z_{3<h_{-1}>}(0.4), z_{3<h_{0}>}(0.6)\right\}$ & $\left\{z_{2<h_{-2}>}(0.3), z_{2<h_{1}>}(0.5), z_{3<h_{-1}>}(0.2)\right\}$ \\
\hline $\mathrm{DP}_{4}$ & $\left\{z_{-3<h_{1}>}(0.5), z_{-3<h_{2}>}(0.3), z_{-2<h_{1}>}(0.2)\right\}$ & $\left\{z_{-2<h_{0}>}(0.5), z_{-1<h_{1}>}(0.2), z_{-1<h_{2}>}(0.3)\right\}$ \\
\hline $\mathrm{DP}_{5}$ & $\left\{z_{-2<h_{1}>}(0.4), z_{0<h_{0}>}(0.4), z_{2<h_{-2}>}(0.2)\right\}$ & $\left\{z_{3<h_{-2}>}(0.2), z_{3<h_{-1}>}(0.2), z_{3<h_{0}>}(0.6)\right\}$ \\
\hline
\end{tabular}

Now, the built PDHL-EDAS method is used to select the optimal 3D printer.

Step 1. Transform the cost indicators into benefit indicators and derive the normalized decision matrix which are shown in Tables 4-6.

Table 4. The normalized PDHLTSs evaluation of each alternative is provided by $\mathrm{e}_{1}$

\begin{tabular}{|l|l|l|}
\hline & \multicolumn{1}{|c|}{$\mathrm{AT}_{1}$} & \multicolumn{1}{c|}{$\mathrm{AT}_{2}$} \\
\hline $\mathrm{DP}_{1}$ & $\left\{z_{-3<h_{0}>}(0.6), z_{-2<h_{1}>}(0.3), z_{-2<h_{2}>}(0.1)\right\}$ & $\left\{z_{-3<h_{2}>}(0.2), z_{-2<h_{0}>}(0.5), z_{-1<h_{2}>}(0.3)\right\}$ \\
\hline $\mathrm{DP}_{2}$ & $\left\{z_{3<h_{-1}>}(0.0), z_{3<h_{-1}>}(0.1), z_{3<h_{0}>}(0.9)\right\}$ & $\left\{z_{3<h_{0}>}(0.0), z_{3<h_{0}>}(0.0), z_{3<h_{0}>}(1.0)\right\}$ \\
\hline $\mathrm{DP}_{3}$ & $\left\{z_{0<h_{1}>}(0.3), z_{1<h_{0}>}(0.4), z_{2<h_{1}>}(0.3)\right\}$ & $\left\{z_{1<h_{2}>}(0.5), z_{2<h_{0}>}(0.3), z_{3<h_{0}>}(0.2)\right\}$ \\
\hline $\mathrm{DP}_{4}$ & $\left\{z_{-3<h_{2}>}(0.4), z_{-2<h_{1}>}(0.3), z_{-1<h_{2}>}(0.3)\right\}$ & $\left\{z_{-3<h_{0}>}(0.5), z_{-2<h_{1}>}(0.2), z_{-1<h_{0}>}(0.3)\right\}$ \\
\hline $\mathrm{DP}_{5}$ & $\left\{z_{-2<h_{0}>}(0.6), z_{-1<h_{0}>}(0.2), z_{0<h_{0}>}(0.2)\right\}$ & $\left\{z_{1<h_{0}>}(0.3), z_{1<h_{2}>}(0.5), z_{2<h_{1}>}(0.2)\right\}$ \\
\hline
\end{tabular}

\begin{tabular}{|l|l|l|}
\hline & \multicolumn{1}{|c|}{$\mathrm{AT}_{3}$} & \multicolumn{1}{c|}{$\mathrm{AT}_{4}$} \\
\hline $\mathrm{DP}_{1}$ & $\left\{z_{-2<h_{0}>}(0.3), z_{-2<h_{1}>}(0.3), z_{-1<h_{2}>}(0.4)\right\}$ & $\left\{z_{-3<h_{0}>}(0.1), z_{-3<h_{2}>}(0.5), z_{-2<h_{2}>}(0.4)\right\}$ \\
\hline $\mathrm{DP}_{2}$ & $\left\{z_{2<h_{0}>}(0.1), z_{3<h_{-1}>}(0.5), z_{3<h_{0}>}(0.4)\right\}$ & $\left\{z_{2<h_{0}>}(0.1), z_{3<h_{-1}>}(0.2), z_{3<h_{0}>}(0.7)\right\}$ \\
\hline $\mathrm{DP}_{3}$ & $\left\{z_{3<h_{-1}>}(0.0), z_{3<h_{-1}>}(0.6), z_{3<h_{0}>}(0.4)\right\}$ & $\left\{z_{1<h_{2}>}(0.7), z_{2<h_{0}>}(0.1), z_{3<h_{0}>}(0.2)\right\}$ \\
\hline $\mathrm{DP}_{4}$ & $\left\{z_{-3<h_{0}>}(0.3), z_{-2<h_{1}>}(0.5), z_{-2<h_{2}>}(0.2)\right\}$ & $\left\{z_{-2<h_{1}>}(0.3), z_{-1<h_{1}>}(0.3), z_{0<h_{1}>}(0.4)\right\}$ \\
\hline $\mathrm{DP}_{5}$ & $\left\{z_{-1<h_{1}>}(0.5), z_{1<h_{0}>}(0.2), z_{2<h_{-2}>}(0.3)\right\}$ & $\left\{z_{3<h_{-2}>}(0.0), z_{3<h_{-2}>}(0.4), z_{3<h_{0}>}(0.6)\right\}$ \\
\hline
\end{tabular}


Table 5. The normalized PDHLTSs evaluation of each alternative is provided by $\mathrm{e}_{2}$

\begin{tabular}{|l|l|l|}
\hline & $\mathrm{AT}_{1}$ & \multicolumn{1}{|c|}{$\mathrm{AT}_{2}$} \\
\hline $\mathrm{DP}_{1}$ & $\left\{z_{-3<h_{0}>}(0.2), z_{-3<h_{2}>}(0.5), z_{-2<h_{1}>}(0.3)\right\}$ & $\left\{z_{-3<h_{1}>}(0.4), z_{-3<h_{2}>}(0.2), z_{-2<h_{1}>}(0.4)\right\}$ \\
\hline $\mathrm{DP}_{2}$ & $\left\{z_{3<h_{0}>}(0.0), z_{3<h_{0}>}(0.0), z_{3<h_{0}>}(1.0)\right\}$ & $\left\{l_{3<s_{-2}>}(0.1), l_{3<s_{-1}>}(0.1), l_{3<s_{0}>}(0.8)\right\}$ \\
\hline $\mathrm{DP}_{3}$ & $\left\{z_{1<h_{1}>}(0.5), z_{2<h_{0}>}(0.3), z_{3<h_{-1}>}(0.2)\right\}$ & $\left\{z_{2<h_{0}>}(0.4), z_{2<h_{-1}>}(0.2), z_{3<h_{-2}>}(0.4)\right\}$ \\
\hline $\mathrm{DP}_{4}$ & $\left\{z_{-3<h_{2}>}(0.3), z_{-2<h_{2}>}(0.4), z_{-1<h_{1}>}(0.3)\right\}$ & $\left\{z_{-3<h_{2}>}(0.6), z_{-2<h_{1}>}(0.2), z_{-2<h_{2}>}(0.2)\right\}$ \\
\hline $\mathrm{DP}_{5}$ & $\left\{z_{1<h_{0}>}(0.5), z_{1<h_{2}>}(0.3), z_{2<h_{1}>}(0.2)\right\}$ & $\left\{z_{0<h_{1}>}(0.7), z_{0<h_{2}>}(0.1), z_{1<h_{1}>}(0.2)\right\}$ \\
\hline
\end{tabular}

\begin{tabular}{|c|l|c|}
\hline & $\mathrm{AT}_{3}$ & $\mathrm{AT}_{4}$ \\
\hline $\mathrm{DP}_{1}$ & $\left\{z_{-2<h_{1}>}(0.2), z_{-2<h_{2}>}(0.5), z_{-1<h_{2}>}(0.3)\right\}$ & $\left\{z_{-3<h_{0}>}(0.7), z_{-2<h_{0}>}(0.2), z_{-2<h_{1}>}(0.1)\right\}$ \\
\hline $\mathrm{DP}_{2}$ & $\left\{z_{3<h_{-1}>}(0.0), z_{3<h_{-1}>}(0.1), z_{3<h_{0}>}(0.9)\right\}$ & $\left\{z_{2<h_{0}>}(0.2), z_{3<h_{-2}>}(0.3), z_{3<h_{0}>}(0.5)\right\}$ \\
\hline $\mathrm{DP}_{3}$ & $\left\{z_{2<h_{0}>}(0.3), z_{3<h_{-2}>}(0.4), z_{3<h_{0}>}(0.3)\right\}$ & $\left\{z_{1<h_{1}>}(0.5), z_{2<h_{0}>}(0.3), z_{2<h_{1}>}(0.2)\right\}$ \\
\hline $\mathrm{DP}_{4}$ & $\left\{z_{-3<h_{1}>}(0.3), z_{-2<h_{0}>}(0.5), z_{-2<h_{1}>}(0.2)\right\}$ & $\left\{z_{-2<h_{1}>}(0.2), z_{-1<h_{0}>}(0.2), z_{-1<h_{2}>}(0.6)\right\}$ \\
\hline $\mathrm{DP}_{5}$ & $\left\{z_{0<h_{1}>}(0.7), z_{1<h_{0}>}(0.2), z_{1<h_{2}>}(0.1)\right\}$ & $\left\{z_{2<h_{0}>}(0.1), z_{3<h_{-1}>}(0.3), z_{3<h_{0}>}(0.6)\right\}$ \\
\hline
\end{tabular}

Table 6. The normalized PDHLTSs evaluation of each alternative is provided by $\mathrm{e}_{3}$

\begin{tabular}{|l|l|c|}
\hline & \multicolumn{1}{|c|}{$\mathrm{AT}_{1}$} & \multicolumn{1}{|c|}{$\mathrm{AT}_{2}$} \\
\hline $\mathrm{DP}_{1}$ & $\left\{z_{-3<h_{2}>}(0.5), z_{-2<h_{0}>}(0.3), z_{-2<h_{1}>}(0.2)\right\}$ & $\left\{z_{-3<h_{0}>}(0.4), z_{-3<h_{1}>}(0.4), z_{-2<h_{0}>}(0.2)\right\}$ \\
\hline $\mathrm{DP}_{2}$ & $\left\{z_{3<h_{0}>}(0.0), z_{3<h_{0}>}(0.0), z_{3<h_{0}>}(1.0)\right\}$ & $\left\{z_{3<h_{-1}>}(0.0), z_{3<h_{-1}>}(0.2), z_{3<h_{0}>}(0.8)\right\}$ \\
\hline $\mathrm{DP}_{3}$ & $\left\{z_{1<h_{2}>}(0.5), z_{2<h_{0}>}(0.3), z_{3<h_{-2}>}(0.2)\right\}$ & $\left\{z_{2<h_{-1}>}(0.4), z_{2<h_{0}>}(0.1), z_{3<h_{-2}>}(0.5)\right\}$ \\
\hline $\mathrm{DP}_{4}$ & $\left\{z_{-3<h_{2}>}(0.1), z_{-2<h_{1}>}(0.6), z_{-1<h_{1}>}(0.3)\right\}$ & $\left\{z_{-3<h_{2}>}(0.7), z_{-2<h_{1}>}(0.2), z_{-2<h_{2}>}(0.1)\right\}$ \\
\hline $\mathrm{DP}_{5}$ & $\left\{z_{-1<h_{0}>}(0.4), z_{0<h_{2}>}(0.4), z_{2<h_{0}>}(0.2)\right\}$ & $\left\{z_{0<h_{1}>}(0.6), z_{0<h_{2}>}(0.3), z_{2<h_{-1}>}(0.1)\right\}$ \\
\hline $\mathrm{AP}_{1}$ & $\left\{z_{-3<h_{2}>}(0.2), z_{-2<h_{0}>}(0.3), z_{-1<h_{2}>}(0.5)\right\}$ & $\left\{z_{-3<h_{0}>}(0.0), z_{-3<h_{0}>}(0.4), z_{-3<h_{1}>}(0.6)\right\}$ \\
\hline $\mathrm{DP}_{2}$ & $\left\{z_{3<h_{-2}>}(0.2), z_{3<h_{-1}>}(0.4), z_{3<h_{0}>}(0.4)\right\}$ & $\left\{z_{3<h_{-2}>}(0.2), z_{3<h_{-1}>}(0.4), z_{3<h_{0}>}(0.4)\right\}$ \\
\hline $\mathrm{DP}_{3}$ & $\left\{z_{3<h_{-1}>}(0.0), z_{3<h_{-1}>}(0.4), z_{3<h_{0}>}(0.6)\right\}$ & $\left\{z_{2<h_{-2}>}(0.3), z_{2<h_{1}>}(0.5), z_{3<h_{-1}>}(0.2)\right\}$ \\
\hline
\end{tabular}


End of Table 6

\begin{tabular}{|c|c|c|}
\hline & $\mathrm{AT}_{3}$ & \multicolumn{1}{c|}{$\mathrm{AT}_{4}$} \\
\hline $\mathrm{DP}_{4}$ & $\left\{z_{-3<h_{1}>}(0.5), z_{-3<h_{2}>}(0.3), z_{-2<h_{1}>}(0.2)\right\}$ & $\left\{z_{-2<h_{0}>}(0.5), z_{-1<h_{1}>}(0.2), z_{-1<h_{2}>}(0.3)\right\}$ \\
\hline $\mathrm{DP}_{5}$ & $\left\{z_{-2<h_{1}>}(0.4), z_{0<h_{0}>}(0.4), z_{2<h_{-2}>}(0.2)\right\}$ & $\left\{z_{3<h_{-2}>}(0.2), z_{3<h_{-1}>}(0.2), z_{3<h_{0}>}(0.6)\right\}$ \\
\hline
\end{tabular}

Step 2. Calculating reference points for all alternatives of each attribute by Eqs (12)-(14), which are shown in Table 7.

Table 7. The Reference points

\begin{tabular}{|c|c|c|}
\hline & $\mathrm{AT}_{1}$ & $\mathrm{AT}_{2}$ \\
\hline $\operatorname{EX}_{1}$ & $\left\{z_{-1<h_{0.13}>}(0.38), z_{-1<h_{0.87}}(0.26), z_{0<h_{0.73}>}(0.36)\right\}$ & $\left\{z_{0<h_{0.07}>}(0.30), z_{0<h_{0.60}}(0.30), z_{1<h_{0.40}>}(0.40)\right\}$ \\
\hline $\operatorname{EX}_{2}$ & $\left\{z_{0<h_{0.00}>}(0.30), z_{0<h_{0.60}>}(0.30), z_{1<h_{0.13}>}(0.40)\right\}$ & $\left\{z_{-1<h_{0.87}>}(0.46), z_{0<h_{0.27}>}(0.14), z_{0<h_{0.73}>}(0.40)\right\}$ \\
\hline $\operatorname{EX}_{3}$ & $\left\{z_{-1<h_{0.80}>}(0.30), z_{0<h_{0.40}>}(0.32), z_{1<h_{0.06}>}(0.38)\right\}$ & $\left\{z_{-1<h_{0.87}>}(0.42), z_{0<h_{0.20}>}(0.22), z_{0<h_{0.73}>}(0.36)\right\}$ \\
\hline
\end{tabular}

\begin{tabular}{|c|c|c|}
\hline & $\mathrm{AT}_{3}$ & $\mathrm{AT}_{4}$ \\
\hline $\operatorname{EX}_{1}$ & $\left\{z_{-1<h_{0.80}>}(0.24), z_{0<h_{0.67}>}(0.42), z_{1<h_{0.20}>}(0.34)\right\}$ & $\left\{z_{0<h_{0.27}>}(0.24), z_{0<h_{0.80}>}(0.30), z_{1<h_{0.47}>}(0.46)\right\}$ \\
\hline $\operatorname{EX}_{2}$ & $\left\{z_{0<h_{0.13}>}(0.30), z_{0<h_{0.53}>}(0.34), z_{1<h_{0.13}>}(0.36)\right\}$ & $\left\{z_{0<h_{0.13}>}(0.34), z_{0<h_{0.80}>}(0.26), z_{1<h_{0.27}>}(0.38)\right\}$ \\
\hline $\operatorname{EX}_{3}$ & $\left\{z_{-1<h_{0.67}>}(0.26), z_{0<h_{0.20}>}(0.36), z_{1<h_{0.07}>}(0.38)\right\}$ & $\left\{z_{0<h_{0.20}>}(0.24), z_{0<h_{0.80}>}(0.34), z_{1<h_{0.13}>}(0.42)\right\}$ \\
\hline
\end{tabular}

Step 3. Calculating the PDHLTS prospect weight of each alternative by Eqs (15)-(16) which are shown in Tables 8-10.

Table 8. The prospect weight of each alternative based on $\mathrm{e}_{1}$

\begin{tabular}{|c|c|c|c|c|}
\hline & $\mathrm{AT}_{1}$ & $\mathrm{AT}_{2}$ & $\mathrm{AT}_{3}$ & $\mathrm{AT}_{4}$ \\
\hline $\mathrm{DP}_{1}$ & 0.1732 & 0.1685 & 0.1812 & 0.1596 \\
\hline $\mathrm{DP}_{2}$ & 0.2377 & 0.2371 & 0.2259 & 0.2163 \\
\hline $\mathrm{DP}_{3}$ & 0.2168 & 0.2170 & 0.2319 & 0.2252 \\
\hline $\mathrm{DP}_{4}$ & 0.1852 & 0.1614 & 0.1719 & 0.1777 \\
\hline $\mathrm{DP}_{5}$ & 0.1871 & 0.2161 & 0.1891 & 0.2211 \\
\hline
\end{tabular}

Table 9. The prospect weight of each alternative based on $\mathrm{e}_{2}$

\begin{tabular}{|c|c|c|c|c|}
\hline & $\mathrm{AT}_{1}$ & $\mathrm{AT}_{2}$ & $\mathrm{AT}_{3}$ & $\mathrm{AT}_{4}$ \\
\hline $\mathrm{DP}_{1}$ & 0.1611 & 0.1685 & 0.1857 & 0.1583 \\
\hline $\mathrm{DP}_{2}$ & 0.2372 & 0.2293 & 0.2460 & 0.2183 \\
\hline $\mathrm{DP}_{3}$ & 0.2156 & 0.2154 & 0.2253 & 0.2189 \\
\hline $\mathrm{DP}_{4}$ & 0.1715 & 0.1711 & 0.1719 & 0.1795 \\
\hline $\mathrm{DP}_{5}$ & 0.2145 & 0.2156 & 0.1712 & 0.2250 \\
\hline
\end{tabular}


Table 10. The prospect weight of each alternative based on $e_{3}$

\begin{tabular}{|c|c|c|c|c|}
\hline & $\mathrm{AT}_{1}$ & $\mathrm{AT}_{2}$ & $\mathrm{AT}_{3}$ & $\mathrm{AT}_{4}$ \\
\hline $\mathrm{DP}_{1}$ & 0.1685 & 0.1622 & 0.1830 & 0.1570 \\
\hline $\mathrm{DP}_{2}$ & 0.2423 & 0.2345 & 0.2235 & 0.2202 \\
\hline $\mathrm{DP}_{3}$ & 0.2210 & 0.2173 & 0.2320 & 0.2235 \\
\hline $\mathrm{DP}_{4}$ & 0.1784 & 0.1693 & 0.1682 & 0.1769 \\
\hline $\mathrm{DP}_{5}$ & 0.1899 & 0.2167 & 0.1934 & 0.2224 \\
\hline
\end{tabular}

Step 4. Calculating The PDHLTSs decision information with prospects weight of each alternative based on each expert by Eq. (17). And assume that the decision information given by each expert is of the same significance, then got overall decision matrix which are shown in Tables 11-14.

Table 11. The PDHLTSs decision information with prospects weight based on $\mathrm{e}_{1}$

\begin{tabular}{|c|l|l|}
\hline & \multicolumn{1}{|c|}{$\mathrm{AT}_{1}$} & \multicolumn{1}{c|}{$\mathrm{AT}_{2}$} \\
\hline $\mathrm{DP}_{1}$ & $\left\{z_{-2.4803<h_{1.5590}}(0.1732)\right\}$ & $\left\{z_{-2.2700<h_{2.1899}}(0.1685)\right\}$ \\
\hline $\mathrm{DP}_{2}$ & $\left\{z_{1.1209<h_{0.3627}}(0.2377)\right\}$ & $\left\{z_{1.2678<h_{0.8035}}(0.2371)\right\}$ \\
\hline $\mathrm{DP}_{3}$ & $\left\{z_{-0.2545<h_{2.2365}}(0.2168)\right\}$ & $\left\{z_{0.3995<h_{1.1984}}(0.2170)\right\}$ \\
\hline $\mathrm{DP}_{4}$ & $\left\{z_{-2.1356<h_{2.5931}}(0.1852)\right\}$ & $\left\{z_{-2.4620<h_{1.6140}}(0.1614)\right\}$ \\
\hline $\mathrm{DP}_{5}$ & $\left\{z_{-1.8776<h_{0.3671}}(0.1871)\right\}$ & $\left\{z_{0.0247<h_{0.0742}>}(0.2161)\right\}$ \\
\hline
\end{tabular}

\begin{tabular}{|c|l|l|}
\hline & \multicolumn{1}{|c|}{$\mathrm{AT}_{3}$} & \multicolumn{1}{c|}{$\mathrm{AT}_{4}$} \\
\hline $\mathrm{DP}_{1}$ & $\left\{z_{-2.0941<h_{2.7176}}(0.1812)\right\}$ & $\left\{z_{-2.6275<h_{1.1175}}(0.1596)\right\}$ \\
\hline $\mathrm{DP}_{2}$ & $\left\{z_{0.7645<h_{2.2936}>}(0.2259)\right\}$ & $\left\{z_{0.6044<h_{1.8133}>}(0.2163)\right\}$ \\
\hline $\mathrm{DP}_{3}$ & $\left\{z_{1.0198<h_{0.0595}>}(0.2319)\right\}$ & $\left\{z_{0.3787<h_{1.1362}>}(0.2252)\right\}$ \\
\hline $\mathrm{DP}_{4}$ & $\left\{z_{-2.4269<h_{1.7194}}(0.1719)\right\}$ & $\left\{z_{-1.7558<h_{0.7327}}(0.1777)\right\}$ \\
\hline $\mathrm{DP}_{5}$ & $\left\{z_{-0.9198<h_{0.2405}>}(0.1891)\right\}$ & $\left\{z_{0.6849<h_{2.0546}>}(0.2211)\right\}$ \\
\hline
\end{tabular}


Table 12. The PDHLTSs decision information with prospects weight based on $\mathrm{e}_{2}$

\begin{tabular}{|c|l|l|}
\hline & \multicolumn{1}{|c|}{$\mathrm{AT}_{1}$} & \multicolumn{1}{c|}{$\mathrm{AT}_{2}$} \\
\hline $\mathrm{DP}_{1}$ & $\left\{z_{-2.6778<h_{0.9666}}(0.1611)\right\}$ & $\left\{z_{-2.6067<h_{1.1798}}(0.1685)\right\}$ \\
\hline $\mathrm{DP}_{2}$ & $\left\{z_{1.2698<h_{0.8094}}(0.2372)\right\}$ & $\left\{z_{0.8979<h_{2.6937}>}(0.2293)\right\}$ \\
\hline $\mathrm{DP}_{3}$ & $\left\{z_{0.2347<h_{0.7041}>}(0.2156)\right\}$ & $\left\{z_{0.2315<h_{0.6945}>}(0.2154)\right\}$ \\
\hline $\mathrm{DP}_{4}$ & $\left\{z_{-2.1996<h_{2.4013}}(0.1715)\right\}$ & $\left\{z_{-2.3727<h_{1.8820}}(0.1711)\right\}$ \\
\hline $\mathrm{DP}_{5}$ & $\left\{z_{0.0032<h_{0.0096}>}(0.2145)\right\}$ & $\left\{z_{-0.5561<h_{1.3317}>}(0.2156)\right\}$ \\
\hline
\end{tabular}

\begin{tabular}{|c|l|l|}
\hline & \multicolumn{1}{|c|}{$\mathrm{AT}_{3}$} & \multicolumn{1}{c|}{$\mathrm{AT}_{4}$} \\
\hline $\mathrm{DP}_{1}$ & $\left\{z_{-1.9478<h_{0.1567}}(0.1857)\right\}$ & $\left\{z_{-2.6307<h_{1.1079}}(0.1583)\right\}$ \\
\hline $\mathrm{DP}_{2}$ & $\left\{z_{1.2641<h_{0.7923}}(0.2460)\right\}$ & $\left\{z_{0.5655<h_{1.6965}}(0.2183)\right\}$ \\
\hline $\mathrm{DP}_{3}$ & $\left\{z_{0.6792<h_{2.0375}}(0.2253)\right\}$ & $\left\{z_{0.2111<h_{0.6334}}(0.2189)\right\}$ \\
\hline $\mathrm{DP}_{4}$ & $\left\{z_{-2.5417<h_{1.3750}}(0.1719)\right\}$ & $\left\{z_{-1.9232<h_{0.2304}}>\right.$ \\
\hline $\mathrm{DP}_{5}$ & $\left\{z_{-0.9458<h_{0.1625}>}(0.1712)\right\}$ & $\left\{z_{0.7504<h_{2.2511}>}(0.2250)\right\}$ \\
\hline
\end{tabular}

Table 13. The PDHLTSs decision information with prospects weight based on $\mathrm{e}_{3}$

\begin{tabular}{|c|l|l|}
\hline & \multicolumn{1}{|c|}{$\mathrm{AT}_{1}$} & \multicolumn{1}{c|}{$\mathrm{AT}_{2}$} \\
\hline $\mathrm{DP}_{1}$ & $\left\{z_{-2.4946<h_{1.5161}}(0.1685)\right\}$ & $\left\{z_{-2.7837<h_{0.6488}}(0.1622)\right\}$ \\
\hline $\mathrm{DP}_{2}$ & $\left\{z_{1.3606<h_{1.0818}}(0.2423)\right\}$ & $\left\{z_{1.0645<h_{0.1934}}(0.2345)\right\}$ \\
\hline $\mathrm{DP}_{3}$ & $\left\{z_{0.3148<h_{0.9445}>}(0.2210)\right\}$ & $\left\{z_{0.2601<h_{0.7804}}(0.2173)\right\}$ \\
\hline $\mathrm{DP}_{4}$ & $\left\{z_{-2.1676<h_{2.4973}}(0.1784)\right\}$ & $\left\{z_{-2.3792<h_{1.8623}}(0.1693)\right\}$ \\
\hline $\mathrm{DP}_{5}$ & $\left\{z_{-0.9741<h_{0.0776}}(0.1899)\right\}$ & $\left\{z_{-0.4723<h_{1.5830}}>\right.$ \\
\hline
\end{tabular}

\begin{tabular}{|c|l|l|}
\hline & \multicolumn{1}{|c|}{$\mathrm{AT}_{3}$} & \multicolumn{1}{c|}{$\mathrm{AT}_{4}$} \\
\hline $\mathrm{DP}_{1}$ & $\left\{z_{-2.069<h_{2.3794}>}(0.1830)\right\}$ & $\left\{z_{-2.9477<h_{0.1570}>}(0.1570)\right\}$ \\
\hline $\mathrm{DP}_{2}$ & $\left\{z_{0.7989<h_{2.3966}>}(0.2235)\right\}$ & $\left\{z_{0.7432<h_{2.2297}>}(0.2202)\right\}$ \\
\hline
\end{tabular}


End of Table 13

\begin{tabular}{|c|l|l|}
\hline & \multicolumn{1}{|c|}{$\mathrm{AT}_{3}$} & \multicolumn{1}{c|}{$\mathrm{AT}_{4}$} \\
\hline $\mathrm{DP}_{3}$ & $\left\{z_{1.0207<h_{0.0620}>}(0.2320)\right\}$ & $\left\{z_{0.4270<h_{1.2811}>}(0.2235)\right\}$ \\
\hline $\mathrm{DP}_{4}$ & $\left\{z_{-2.6076<h_{1.1772}}(0.1682)\right\}$ & $\left\{z_{-1.9387<h_{0.1838}>}(0.1769)\right\}$ \\
\hline $\mathrm{DP}_{5}$ & $\left\{z_{-1.3241<h_{2.0278}}(0.1934)\right\}$ & $\left\{z_{0.7812<h_{2.3436}>}(0.2224)\right\}$ \\
\hline
\end{tabular}

Table 14. The overall decision matrix

\begin{tabular}{|c|c|c|}
\hline & $\mathrm{AT}_{1}$ & $\mathrm{AT}_{2}$ \\
\hline $\mathrm{DP}_{1}$ & $\left\{\begin{array}{l}\left\{z_{-2.6778<h_{0.9666}>}(0.1611)\right\},\left\{z_{-2.4946<h_{1.5161}>}(0.1685)\right\}, \\
\left\{z_{-2.4803<h_{1.5590}}(0.1732)\right\}\end{array}\right\}$ & $\left\{\begin{array}{l}\left\{z_{-2.7837<h_{0.6488}>}(0.1622)\right\},\left\{z_{-2.6067<h_{1.1798}>}(0.1685)\right\} \\
\left\{z_{-2.2700<h_{2.1899}>}(0.1685)\right\}\end{array}\right.$ \\
\hline $\mathrm{DP}_{2}$ & 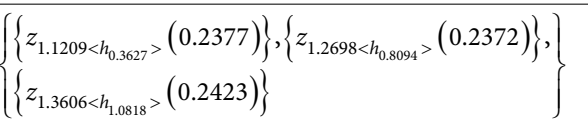 & $\left\{\begin{array}{l}\left\{z_{0.8979<h_{2.6937}>}(0.2293)\right\},\left\{z_{1.0645<h_{0.1934}>}(0.2345)\right\}, \\
\left\{z_{1.2678<h_{0.8035}>}(0.2371)\right\}\end{array}\right\}$ \\
\hline $\mathrm{DP}_{3}$ & 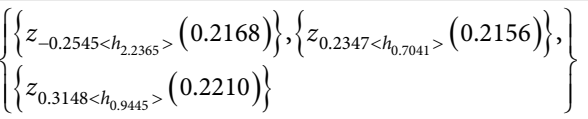 & $\left\{\begin{array}{l}\left\{z_{0.2315<h_{0.6945}>}(0.2154)\right\},\left\{z_{0.2601<h_{0.7804}>}(0.2173)\right\}, \\
\left\{z_{0.3995<h_{1.1984}>}(0.2170)\right\}\end{array}\right\}$ \\
\hline $\mathrm{DP}_{4}$ & $\left\{\begin{array}{l}\left\{z_{-2.1996<h_{2.4013}>}(0.1715)\right\},\left\{z_{-2.1676<h_{2.4973}>}(0.1784)\right\}, \\
\left\{z_{-2.1356<h_{2.5931}>}(0.1852)\right\}\end{array}\right\}$ & $\left\{\begin{array}{l}\left\{z_{-2.4620<h_{1.6140}>}(0.1614)\right\},\left\{z_{-2.3792<h_{1.8623}>}(0.1693)\right\}, \\
\left\{z_{-2.3727<h_{1.8220}>}(0.1711)\right\}\end{array}\right.$ \\
\hline $\mathrm{DP}_{5}$ & $\left\{\begin{array}{l}\left\{z_{-1.8776<h_{0.3671}>}(0.1871)\right\},\left\{z_{-0.9741<h_{0.0776}>}(0.1899)\right\}, \\
\left\{z_{0.0032<h_{0.0096}>}(0.2145)\right\}\end{array}\right\}$ & $\left\{\begin{array}{l}\left\{z_{-0.5561<h_{1.3317}>}(0.2156)\right\},\left\{z_{-0.4723<h_{1.5830}>}(0.2167)\right\}, \\
\left\{z_{0.0247<h_{0.0742}>}(0.2161)\right\}\end{array}\right\}$ \\
\hline
\end{tabular}

\begin{tabular}{|c|c|c|}
\hline & $\mathrm{AT}_{3}$ & $\mathrm{AT}_{4}$ \\
\hline $\mathrm{DP}_{1}$ & $\left\{\begin{array}{l}\left\{z_{-2.0941<h_{2.7176}>}(0.1812)\right\},\left\{z_{-2.069<h_{2.3794}>}(0.1830)\right\}, \\
\left\{z_{-1.9478<h_{0.1567}>}(0.1857)\right\}\end{array}\right\}$ & $\left\{\begin{array}{l}\left\{z_{-2.9477<h_{0.1570}>}(0.1570)\right\},\left\{z_{-2.6307<h_{1.1079}>}(0.1583)\right\}, \\
\left\{z_{-2.6275<h_{1.1175}>}(0.1596)\right\}\end{array}\right.$ \\
\hline $\mathrm{DP}_{2}$ & 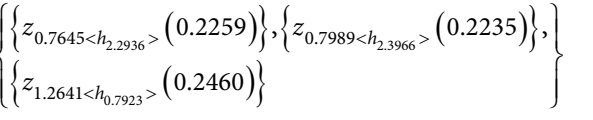 & $\left\{\begin{array}{l}\left\{z_{0.5655<h_{1.6965}>}(0.2183)\right\},\left\{z_{0.6044<h_{1.8133}>}(0.2163)\right\}, \\
\left\{z_{0.7432<h_{2.2297}>}(0.2202)\right\}\end{array}\right\}$ \\
\hline $\mathrm{DP}_{3}$ & 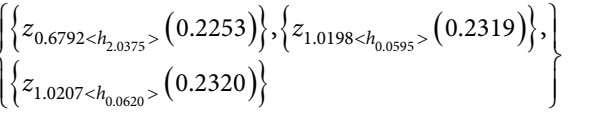 & 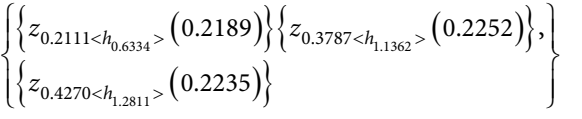 \\
\hline $\mathrm{DP}_{4}$ & $\left\{\begin{array}{l}\left\{z_{-2.6076<h_{1.1772}>}(0.1682)\right\},\left\{z_{-2.5417<h_{1.3750}>}(0.1719)\right\}, \\
\left\{z_{-2.4269<h_{1.7194}>}(0.1719)\right\}\end{array}\right\}$ & $\left\{\begin{array}{l}\left\{z_{-1.9387<h_{0.1838}>}(0.1769)\right\},\left\{z_{-1.9232<h_{0.2304}>}(0.1615)\right\}, \\
\left\{z_{-1.7558<h_{0.7327}>}(0.1777)\right\}\end{array}\right.$ \\
\hline $\mathrm{DP}_{5}$ & $\left\{\begin{array}{l}\left\{z_{-1.3241<h_{2.0278}>}(0.1934)\right\},\left\{z_{-0.9458<h_{0.1625}>}(0.1712)\right\}, \\
\left\{z_{-0.9198<h_{0.2405}>}(0.1891)\right\}\end{array}\right\}$ & $\left\{\begin{array}{l}\left\{z_{0.6849<h_{2.0546}>}(0.2211)\right\},\left\{z_{0.7504<h_{2.251>}>}(0.2250)\right\}, \\
\left\{z_{0.7812<h_{2.3436}>}(0.2224)\right\}\end{array}\right\}$ \\
\hline
\end{tabular}


Step 5. The normalized overall decision matrix could be obtained with Eq. (5) and shown in Table 15.

Table 15. The normalized overall decision matrix

\begin{tabular}{|c|c|c|}
\hline & $\mathrm{AT}_{1}$ & $\mathrm{AT}_{2}$ \\
\hline $\mathrm{DP}_{1}$ & $\left\{\begin{array}{l}\left\{z_{-2.6778<h_{0.9666}>}(0.3204)\right\},\left\{z_{-2.4946<h_{1.5161}>}(0.3350)\right\}, \\
\left\{z_{-2.4803<h_{1.5590}>}(0.3445)\right\}\end{array}\right\}$ & $\left\{\begin{array}{l}\left\{z_{-2.7837<h_{0.6488}>}(0.3249)\right\},\left\{z_{-2.6067<h_{1.1798}>}(0.3376)\right\}, \\
\left\{z_{-2.2700<h_{2.1899}>}(0.3374)\right\}\end{array}\right\}$ \\
\hline $\mathrm{DP}_{2}$ & $\left\{\begin{array}{l}\left\{z_{1.1209<h_{0.3627}>}(0.3315)\right\},\left\{z_{1.2698<h_{0.8094}>}(0.3307)\right\}, \\
\left\{z_{1.3606<h_{1.0818}>}(0.3378)\right\}\end{array}\right\}$ & $\left\{\begin{array}{l}\left\{z_{0.8979<h_{2.6937}>}(0.3271)\right\},\left\{z_{1.0645<h_{0.1934}>}(0.3346)\right\}, \\
\left\{z_{1.2678<h_{0.8035}>}(0.3383)\right\}\end{array}\right\}$ \\
\hline $\mathrm{DP}_{3}$ & $\left\{\begin{array}{l}\left\{z_{-0.2545<h_{2.2365}>}(0.3317)\right\},\left\{z_{0.2347<h_{0.7041}>}(0.3300)\right\}, \\
\left\{z_{0.3148<h_{0.9445}>}(0.3382)\right\}\end{array}\right\}$ & $\left\{\begin{array}{l}\left\{z_{0.2315<h_{0.6945}>}(0.3316)\right\},\left\{z_{0.2601<h_{0.7804}>}(0.3345)\right\}, \\
\left\{z_{0.3995<h_{1.1984}>}(0.3339)\right\}\end{array}\right\}$ \\
\hline $\mathrm{DP}_{4}$ & $\left\{\begin{array}{l}\left\{z_{-2.1996<h_{2.4013}>}(0.3205)\right\},\left\{z_{-2.1676<h_{2.4973}>}(0.3333)\right\}, \\
\left\{z_{-2.1356<h_{2.5931}>}(0.3461)\right\}\end{array}\right\}$ & $\left\{\begin{array}{l}\left\{z_{-2.4620<h_{1.6140}>}(0.3217)\right\},\left\{z_{-2.3792<h_{1.8623}>}(0.3374)\right\}, \\
\left\{z_{-2.3727<h_{1.8820}>}(0.3410)\right\},\end{array}\right.$ \\
\hline $\mathrm{DP}_{5}$ & $\left\{\begin{array}{l}\left\{z_{-1.8776<h_{0.3671}>}(0.3162)\right\},\left\{z_{-0.9741<h_{0.0776}>}(0.3211)\right\}, \\
\left\{z_{0.0032<h_{0.0096}>}(0.3627)\right\}\end{array}\right\}$ & $\left\{\begin{array}{l}\left\{z_{-0.5561<h_{1.3317}>}(0.3326)\right\},\left\{z_{-0.4723<h_{1.5830}>}(0.3342)\right\}, \\
\left\{z_{0.0247<h_{0.0742}>}(0.3332)\right\}\end{array}\right\}$ \\
\hline
\end{tabular}

\begin{tabular}{|c|c|c|}
\hline & $\mathrm{AT}_{3}$ & $\mathrm{AT}_{4}$ \\
\hline $\mathrm{DP}_{1}$ & $\left\{\begin{array}{l}\left\{z_{-2.0941<h_{2.7176}>}(0.3314)\right\},\left\{z_{-2.069<h_{2.3794}>}(0.3303)\right\}, \\
\left\{z_{-1.9478<h_{0.1567}>}(0.3383)\right\}\end{array}\right\}$ & $\left\{\begin{array}{l}\left\{z_{-2.9477<h_{0.1570}>}(0.3306)\right\},\left\{z_{-2.6307<h_{1.1079}>}(0.3333)\right\}, \\
\left\{z_{-2.6275<h_{1.1175}>}(0.3362)\right\}\end{array}\right\}$ \\
\hline $\mathrm{DP}_{2}$ & $\left\{\begin{array}{l}\left\{z_{0.7645<h_{2.2936}>}(0.3248)\right\},\left\{z_{0.7989<h_{2.3966}>}(0.3214)\right\}, \\
\left\{z_{1.2641<h_{0.7923}>}(0.3538)\right\}\end{array}\right\}$ & $\left\{\begin{array}{l}\left\{z_{0.5655<h_{1.6965}>}(0.3334)\right\},\left\{z_{0.6044<h_{1.8133}>}(0.3303)\right\}, \\
\left\{z_{0.7432<h_{2.2297}>}(0.3363)\right\}\end{array}\right\}$ \\
\hline $\mathrm{DP}_{3}$ & $\left\{\begin{array}{l}\left\{z_{0.6792<h_{2.0375}>}(0.3269)\right\},\left\{z_{1.0198<h_{0.0595}>}(0.3365)\right\}, \\
\left\{z_{1.0207<h_{0.0620}>}(0.3366)\right\}\end{array}\right\}$ & $\left\{\begin{array}{l}\left\{z_{0.2111<h_{0.6334}>}(0.3279)\right\}\left\{z_{0.3787<h_{1.1362}>}(0.3374)\right\}, \\
\left\{z_{0.4270<h_{1.2811}>}(0.3347)\right\}\end{array}\right\}$ \\
\hline $\mathrm{DP}_{4}$ & $\left\{\begin{array}{l}\left\{z_{-2.6076<h_{1.1772}>}(0.3285)\right\},\left\{z_{-2.5417<h_{1.3750}>}(0.3357)\right\}, \\
\left\{z_{-2.4269<h_{1.7194}>}(0.3358)\right\}\end{array}\right\}$ & $\left\{\begin{array}{l}\left\{z_{-1.9387<h_{0.1838}>}(0.3427)\right\},\left\{z_{-1.9232<h_{0.2304}>}(0.3129)\right\}, \\
\left\{z_{-1.7558<h_{0.7327}>}(0.3444)\right\}\end{array}\right.$ \\
\hline $\mathrm{DP}_{5}$ & $\left\{\begin{array}{l}\left\{z_{-1.3241<h_{2.0278}>}(0.3493)\right\},\left\{z_{-0.9458<h_{0.1625}>}(0.3092)\right\}, \\
\left\{z_{-0.9198<h_{0.2405}>}(0.3416)\right\}\end{array}\right\}$ & $\left\{\begin{array}{l}\left\{\begin{array}{l}z_{0.6849<h_{2.0546}>}(0.3307) \\
\left\{z_{0.7812<h_{2.3436}>}(0.3327)\right.\end{array}\right\},\left\{z_{0.7504<h_{2.2511}>}(0.3366)\right\},\end{array}\right\}$ \\
\hline
\end{tabular}

Step 6. Through CRITIC method get the objective weight of attributes is $o \omega=(0.1988,0.1248$, $0.2518,0.4246)$. Therefore, the combine weight of attribute is $c \omega_{j}=(0.2391,0.1601$, $0.2330,0.3677)$.

Step 7. The probabilistic double hierarchy linguistic average values are competed by Eqs (23)-(25) shown in Table 16. 
Table 16. NPWAVPDHL for all attributes

\begin{tabular}{|c|l|}
\hline & PDHLBBA \\
\hline $\mathrm{AT}_{1}$ & $\left\{z_{-0.7554<h_{0.2446}>}(0.3241), z_{-0.4527<h_{0.5473}>}(0.3301), z_{-0.1749<h_{0.8251}>}(0.3459)\right\}$ \\
\hline $\mathrm{AT}_{2}$ & $\left\{z_{-0.4690<h_{0.5310}>}(0.3276), z_{-0.4535<h_{0.5465}>}(0.3356), z_{-0.1803<h_{0.8197}>}(0.3368)\right\}$ \\
\hline $\mathrm{AT}_{3}$ & $\left\{z_{-0.2799<h_{0.7201}>}(0.3322), z_{-0.3050<h_{0.6950}>}(0.3266), z_{-0.4039<h_{0.5961}>}(0.3412)\right\}$ \\
\hline $\mathrm{AT}_{4}$ & $\left\{z_{-0.3700<h_{0.6300}>}(0.3331), z_{-0.1281<h_{0.8719}>}(0.3301), z_{0.0273<h_{0.0273}>}(0.3369)\right\}$ \\
\hline
\end{tabular}

Step 8. The NPWPDHLPDA can be calculated by Eq. (26), and the NPWPDHLNDA can be calculated by Eq. (27) which are shown in Tables 17-18.

Table 17. The NPWPDHLPDA matrix

\begin{tabular}{|c|c|c|c|c|}
\hline & $\mathrm{AT}_{1}$ & $\mathrm{AT}_{2}$ & $\mathrm{AT}_{3}$ & $\mathrm{AT}_{4}$ \\
\hline $\mathrm{DP}_{1}$ & 0.0000 & 0.0000 & 0.0000 & 0.0000 \\
\hline $\mathrm{DP}_{2}$ & 0.7689 & 0.7030 & 0.7047 & 0.5038 \\
\hline $\mathrm{DP}_{3}$ & 0.3872 & 0.3649 & 0.5524 & 0.2941 \\
\hline $\mathrm{DP}_{4}$ & 0.0000 & 0.0000 & 0.0000 & 0.0000 \\
\hline $\mathrm{DP}_{5}$ & 0.0000 & 0.1383 & 0.0000 & 0.5747 \\
\hline
\end{tabular}

Table 18. NPWPDHLNDA matrix

\begin{tabular}{|c|c|c|c|c|}
\hline & $\mathrm{AT}_{1}$ & $\mathrm{AT}_{2}$ & $\mathrm{AT}_{3}$ & $\mathrm{AT}_{4}$ \\
\hline $\mathrm{DP}_{1}$ & 0.6451 & 0.6587 & 0.4401 & 0.8132 \\
\hline $\mathrm{DP}_{2}$ & 0.0000 & 0.0000 & 0.0000 & 0.0000 \\
\hline $\mathrm{DP}_{3}$ & 0.0000 & 0.0000 & 0.0000 & 0.0000 \\
\hline $\mathrm{DP}_{4}$ & 0.3453 & 0.5471 & 0.6440 & 0.5575 \\
\hline $\mathrm{DP}_{5}$ & 0.1581 & 0.0000 & 0.1717 & 0.0000 \\
\hline
\end{tabular}

Step 9. Calculating the weight sum of NPWPDHLPDA and NPWPDHLNDA of all given alternatives. And their normalized values by Eqs (32)-(35) shown in Table 19.

Table 19. NPWPDHL(SP/NSP) and NPWPDHL(SN/NSN) matrix

\begin{tabular}{|c|c|c|c|c|}
\hline & NPWPDHL(SP) & NPWPDHL(NSP) & NPWPDHL(SN) & NPWPDHL(NSN) \\
\hline $\mathrm{DP}_{1}$ & 0.0000 & 0.0000 & 0.6613 & 0.0000 \\
\hline $\mathrm{DP}_{2}$ & 0.6459 & 1.0000 & 0.0000 & 1.0000 \\
\hline $\mathrm{DP}_{3}$ & 0.3879 & 0.6005 & 0.0000 & 1.0000 \\
\hline $\mathrm{DP}_{4}$ & 0.0000 & 0.0000 & 0.5252 & 0.2058 \\
\hline $\mathrm{DP}_{5}$ & 0.2335 & 0.3615 & 0.0778 & 0.8823 \\
\hline
\end{tabular}


Step 10. Calculated the assessment value matrix by Eq. (36) shown in Table 20.

Table 20. NPWPDHL(AS) matrix

\begin{tabular}{|c|c|c|c|c|c|}
\hline & $\mathrm{DP}_{1}$ & $\mathrm{DP}_{2}$ & $\mathrm{DP}_{3}$ & $\mathrm{DP}_{4}$ & $\mathrm{DP}_{5}$ \\
\hline$N P W P D H \tilde{L}_{i}^{A S}(\tilde{p})$ & 0.0000 & 1.0000 & 0.8003 & 0.1029 & 0.6219 \\
\hline
\end{tabular}

Step 11. According to the NPWPDH $\tilde{L}_{i}{ }^{A S}(\tilde{p})(i=1,2,3,4,5)$, the order of the given alternatives could be obtained. Evidently, the order is $\mathrm{DP}_{2}>\mathrm{DP}_{3}>\mathrm{DP}_{5}>\mathrm{DP}_{4}>\mathrm{DP}_{1}$ and the most optimal $3 \mathrm{D}$ printer is $\mathrm{DP}_{2}$.

\subsection{Comparative analysis}

Then, The built method is compared with PDHL-VIKOR method (Gou et al., 2020) shown in Table 21.

Table 21. The numerical results and rank derived by the PDHL-VIKOR

\begin{tabular}{|c|c|c|c|c|c|c|}
\hline & PDHLGU & rank & PDHLIK & rank & PDHLC & rank \\
\hline $\mathrm{DP}_{1}$ & 0.9218 & 5 & 0.3645 & 5 & 1.0000 & 5 \\
\hline $\mathrm{DP}_{2}$ & -1.045 & 1 & -0.1693 & 1 & 0.0000 & 1 \\
\hline $\mathrm{DP}_{3}$ & -0.6588 & 2 & -0.0869 & 2 & 0.1754 & 2 \\
\hline $\mathrm{DP}_{4}$ & 0.7200 & 4 & 0.2325 & 4 & 0.8251 & 4 \\
\hline $\mathrm{DP}_{5}$ & -0.3125 & 3 & 0.0674 & 3 & 0.4080 & 3 \\
\hline
\end{tabular}

From Table 21, the ranking of the five alternatives can be obtained as: $\mathrm{DP}_{2}>\mathrm{DP}_{3}>\mathrm{DP}_{5}>$ $\mathrm{DP}_{4}>\mathrm{DP}_{1}$ and the most optimal 3D printer is $\mathrm{DP}_{2}$. Therefore, the PDHL-EDAS method in this paper is scientific and effective.

\section{Conclusions}

The selection of optimal 3D printers is a great significance in the production and sales process of enterprises. Therefore, enterprises urgently need a set of effective decision-making model based on the optimal 3D printer selection problem. Now, a new PDHL-EDAS method for MAGDM is established. Then the feasibility of this method is illustrated by choosing high quality $3 \mathrm{D}$ printing as an example. In addition, the validity and rationality of the developed model is verified, and a comparative analysis is carried out. The contributions of the PDHLEDAS method established in this paper are as follow: (1) the establishment of this method enriches the decision-making method based on PDHLTS; (2) enrich the 3D printer evaluation model.

\section{Acknowledgements}

The work was supported by the Humanities and Social Sciences Foundation of Ministry of Education of the People's Republic of China (No. 15YJCZH138). 


\section{References}

Cheng, Y. L., Qin, H. T., Acevedo, N. C., Jiang, X. P., \& Shi, X. L. (2020). 3D printing of extended-release tablets of theophylline using hydroxypropyl methylcellulose (HPMC) hydrogels. International Journal of Pharmaceutics, 591, 11983. https://doi.org/10.1016/j.ijpharm.2020.119983

Chrispin, T. T. B., Takano, C. C., Fernandes, M. S., \& Sartori, M. G. F. (2020). Development of vaginal devices by 3D printer for gynecological use. International Urogynecology Journal, 31, S52-S53.

Darko, A. P., \& Liang, D. C. (2020). Some q-rung orthopair fuzzy Hamacher aggregation operators and their application to multiple attribute group decision making with modified EDAS method. Engineering Applications of Artificial Intelligence, 87, 103259. https://doi.org/10.1016/j.engappai.2019.103259

Diakoulaki, D., Mavrotas, G., \& Papayannakis, L. (1995). Determining objective weights in multiple criteria problems: The critic method. Computers \& Operations Research, 22(7), 763-770. https://doi.org/10.1016/0305-0548(94)00059-H

Feng, X. Q., Wei, C. P., \& Liu, Q. (2018). EDAS method for extended hesitant fuzzy linguistic multicriteria decision making. International Journal of Fuzzy Systems, 20, 2470-2483.

https://doi.org/10.1007/s40815-018-0504-5

Ghorabaee, M. K., Zavadskas, E. K., Olfat, L., \& Turskis, Z. J. I. (2015). Multi-criteria inventory classification using a new method of Evaluation based on Distance from Average Solution (EDAS). Informatica, 26(3), 435-451. https://doi.org/10.15388/Informatica.2015.57

Gou, X., Liao, H., Xu, Z., \& Herrera, F. (2017). Double hierarchy hesitant fuzzy linguistic term set and MULTIMOORA method: A case of study to evaluate the implementation status of haze controlling measures. Information Fusion, 38, 22-34. https://doi.org/10.1016/j.inffus.2017.02.008

Gou, X., Xu, Z., Liao, H., \& Herrera, F. (2021). Probabilistic double hierarchy linguistic term set and its use in designing an improved VIKOR method: The application in smart healthcare. Journal of the Operational Research Society, 72(12), 2611-2630. https://doi.org/10.1080/01605682.2020.1806741

Gundogdu, F. K., Kahraman, C., \& Civan, H. N. (2018). A novel hesitant fuzzy EDAS method and its application to hospital selection. Journal of Intelligent \& Fuzzy Systems, 35(6), 6353-6365. https://doi.org/10.3233/JIFS-181172

He, Y., Lei, F., Wei, G. W., Wang, R., Wu, J., \& Wei, C. (2019). EDAS method for multiple attribute group decision making with probabilistic uncertain linguistic information and its application to green supplier selection. International Journal of Computational Intelligence Systems, 12(3), 1361-1370. https://doi.org/10.2991/ijcis.d.191028.001

Karunanithi, K., Han, C., Lee, C. J., Shi, W. C., Duan, L., \& Qian, Y. (2015). Identification of a hemodynamic parameter for assessing treatment outcome of EDAS in Moyamoya disease. Journal of Biomechanics, 48(2), 304-309. https://doi.org/10.1016/j.jbiomech.2014.11.029

Kundakci, N. (2019). An integrated method using MACBETH and EDAS methods for evaluating steam boiler alternatives. Journal of Multi-Criteria Decision Analysis, 26(1-2), 27-34. https://doi.org/10.1002/mcda.1656

Lei, F., Wei, G., \& Chen, X. (2021). Model-based evaluation for online shopping platform with probabilistic double hierarchy linguistic CODAS method. International Journal of Intelligent Systems, 36(9), 5339-5358. https://doi.org/10.1002/int.22514

Liang, W. Z., Zhao, G. Y., \& Luo, S. Z. (2018). An integrated EDAS-ELECTRE method with picture fuzzy information for cleaner production evaluation in gold mines. IEEE Access, 6, 65747-65759. https://doi.org/10.1109/ACCESS.2018.2878747

Liu, Y. S., Zhang, R., Ye, H. Q., Wang, S. M., Wang, K. P., Liu, Y. S., \& Zhou, Y. S. (2019). The development of a 3D colour reproduction system of digital impressions with an intraoral scanner and a 3D printer: A preliminary study. Scientific Reports, 9, 20052.

https://doi.org/10.1038/s41598-019-56624-3 
Pang, Q., Wang, H., \& Xu, Z. S. (2016). Probabilistic linguistic linguistic term sets in multi-attribute group decision making. Information Sciences, 369, 128-143. https://doi.org/10.1016/j.ins.2016.06.021

Pavan, L. I., Bourguignon, G. A., Soderini, H., \& Ubertazzi, E. P. (2021). Vaginoplasty: Modificated McIndoe using xenograft and a tailored 3D-printer mold. International Urogynecology Journal, 32, 2283-2285. https://doi.org/10.1007/s00192-021-04689-y

Prabhu, S. R., \& Ilangkumaran, M. (2019a). Decision making methodology for the selection of 3D printer under fuzzy environment. International Journal of Materials \& Product Technology, 59(3), 239-252. https://doi.org/10.1504/IJMPT.2019.102935

Prabhu, S. R., \& Ilangkumaran, M. (2019b). Selection of 3D printer based on FAHP integrated with GRA-TOPSIS. International Journal of Materials \& Product Technology, 58(2/3), 155-177. https://doi.org/10.1504/IJMPT.2019.097667

Stanujkic, D., Zavadskas, E. K., Keshavarz Ghorabaee, M., \& Turskis, Z. (2017). An extension of the EDAS method based on the use of interval grey numbers. Studies in Informatics and Control, 26(1), 5-12. https://doi.org/10.24846/v26ily201701

Wang, P., Wang, J., \& Wei, G. W. (2019). EDAS method for multiple criteria group decision making under 2-tuple linguistic neutrosophic environment. Journal of Intelligent \& Fuzzy Systems, 37(2), 1597-1608. https://doi.org/10.3233/JIFS-179223

Wei, C., Wu, J., Guo, Y., \& Wei, G. (2021a). Green supplier selection based on CODAS method in probabilistic uncertain linguistic environment. Technological and Economic Development of Economy, 27(3), 530-549. https://doi.org/10.3846/tede.2021.14078

Wei, G., Lin, R., Lu, J., Wu, J., \& Wei, C. (2021b). The generalized dice similarity measures for probabilistic uncertain linguistic MAGDM and its application to location planning of electric vehicle charging stations. International Journal of Fuzzy Systems. https://doi.org/10.1007/s40815-021-01084-z

Wei, G., Wei, C., \& Guo, Y. (2021c). EDAS method for probabilistic linguistic multiple attribute group decision making and their application to green supplier selection. Soft Computing, 25, 9045-9053. https://doi.org/10.1007/s00500-021-05842-X

Yi, J. H., Duling, M. G., Bowers, L. N., Knepp, A. K., LeBouf, R. F., Nurkiewicz, T. R., Ranpara, A., Luxton, T., Martin, S. B., Burns, D. A., Peloquin, D. M., Baumann, E. J., Virji, M. A., \& Stefaniak, A. B. (2019). Particle and organic vapor emissions from children's 3-D pen and 3-D printer toys. Inhalation Toxicology, 31(13-14), 432-445. https://doi.org/10.1080/08958378.2019.1705441 